ARTICLE

https://doi.org/10.1038/s41467-020-18139-8

\title{
A lysozyme with altered substrate specificity facilitates prey cell exit by the periplasmic predator Bdellovibrio bacteriovorus
}

Christopher J. Harding (1) 1,3, Simona G. Huwiler (10 2,3, Hannah Somers², Carey Lambert², Luke J. Ray ${ }^{2}$, Rob Till², Georgina Taylor ${ }^{2}$, Patrick J. Moynihan (1) ${ }^{1}$, R. Elizabeth Sockett (i) ${ }^{2 凶}$ \& Andrew L. Lovering (i) ${ }^{1 凶}$

Lysozymes are among the best-characterized enzymes, acting upon the cell wall substrate peptidoglycan. Here, examining the invasive bacterial periplasmic predator Bdellovibrio bacteriovorus, we report a diversified lysozyme, DsIA, which acts, unusually, upon (GIcNAc-) deacetylated peptidoglycan. B. bacteriovorus are known to deacetylate the peptidoglycan of the prey bacterium, generating an important chemical difference between prey and self walls and implying usage of a putative deacetyl-specific "exit enzyme". DslA performs this role, and $\triangle$ DslA strains exhibit a delay in leaving from prey. The structure of DslA reveals a modified lysozyme superfamily fold, with several adaptations. Biochemical assays confirm DslA specificity for deacetylated cell wall, and usage of two glutamate residues for catalysis. Exogenous DslA, added ex vivo, is able to prematurely liberate B. bacteriovorus from prey, part-way through the predatory lifecycle. We define a mechanism for specificity that invokes steric selection, and use the resultant motif to identify wider DsIA homologues.

\footnotetext{
${ }^{1}$ Institute for Microbiology and Infection, School of Biosciences, University of Birmingham, Birmingham B15 2TT, UK. ${ }^{2}$ Medical School, School of Life Sciences, Queen's Medical Centre, University of Nottingham, Nottingham NG7 2UH, UK. ${ }^{3}$ These authors contributed equally: Christopher J. Harding, Simona G. Huwiler.

凶email: liz.sockett@nottingham.ac.uk; a.lovering@bham.ac.uk
} 
T he relationship between lysozyme(s) and the bacterial cell wall is historically prominent in microbiology and biochemistry, starting with the discovery of lysozyme antibacterial activity by Fleming in 1922 (ref. ${ }^{1}$ ) and progressing to the first elucidation of an enzyme structure by Phillips in 1965 (ref. ${ }^{2}$ ). From these beginnings, lysozymes are now among the most highly characterized enzymes in biochemistry. Broadly, the susceptibility of the bacterial cell-wall peptidoglycan to lysozyme activity encompasses the usage of lysozyme in mucosal immunity ${ }^{3}$, pathogen modification of the wall to evade killing ${ }^{4}$, deployment of lysozymes during interbacterial competition ${ }^{5}$, and usage of lysozyme/lytic transglycosylase activity in tailoring the cell wall during growth, division, signaling, autolysis, sporulation, and insertion of wall-spanning protein complexes 6 . Lysozymes (together with related lytic transglycosidases, bracketed as muramidases) cleave peptidoglycan strands between the $\mathrm{C} 1$ of $\mathrm{N}$ acetylmuramic acid (MurNAc) and $\mathrm{C} 4$ of $\mathrm{N}$-acetylglucosamine (GlcNAc) sugars; although modifications of the cell-wall peptide are relatively common, the sugar backbone is much less variable. Modification of peptidoglycan to endow resistance to human lysozyme is common in Gram-positive pathogens which have environmentally exposed cell walls (e.g., Listeria, Streptococcus, Staphylococcus spp.) and takes the form of either O6-acetylation (MurNAc) or N-deacetylation (MurNAc and/or GlcNAc) ${ }^{7}$; both reactions take place on the mature peptidoglycan (PG) polymer. To date, lysozymes with low specificity are known (e.g., cellosyl will turnover both acetylated and deacetylated peptidoglycan $)^{6}$ but no enzyme has been characterized that is specific for the $\mathrm{N}$-deacetylated form only.

The intraperiplasmic bacterial predator Bdellovibrio bacteriovorus is adept at peptidoglycan manipulation, entering through the outer membrane and wall of other Gram-negative bacterial cells to the inner periplasmic compartment and systematically metabolizing bacterial prey cell components from the inside ${ }^{8}$. Other external bacterial predators of bacteria also encode wallmetabolizing enzymes (Myxococcus and Streptomyces among the best characterized), but aside from the endpoint of prey cell destruction, Bdellovibrio differs in that it has entry, residence and exit lifecycle stages that require more nuanced approaches to define and differentially manipulate chemically related self- and bacterial prey cell structures at different timepoints. Exit from prey requires destruction of several complex cell wall and outer membrane structures by predators, without damaging self.

A series of early papers by Thomashow and Rittenberg established that B. bacteriovorus has glycanase and peptidase activities for entry ${ }^{9}$, deacetylase(s) that modify the host cell-wall material ${ }^{10}$, and activities that solubilize/reattach diaminopimelate and acylate the peptidoglycan with a long-chain fatty acid ${ }^{11}$. More recent investigations have characterized some of these enzymes, finding that entry-secreted D,D-endopeptidases from B. bacteriovorus break 3,4 peptide crosslinks in prey PG walls, thereby rounding/ softening prey PG and preventing wasteful entry by a second predator ${ }^{12}$. Bdellovibrio protects itself during these modifications by producing a protein that complexes and inhibits the peptidases - failure to do so results in suicide ${ }^{13}$. Other wall modifications during predation have been observed via the use of fluorescent D-amino acids, revealing a porthole-like structure that forms during an invasion, and other L,D-transpeptidase-dependent activities that reinforce the prey cell "bdelloplast" while occupied by the B. bacteriovorus for its intrabacterial lifecycle ${ }^{14}$.

Initial hypotheses that Bdellovibrio-catalyzed deacetylation of prey peptidoglycan protected against host autolysins were disproven; instead, deacetylation marks prey (as distinct from the predator, which retains acetylated peptidoglycan) for selective destruction at the end of the intraperiplasmic lifecycle ${ }^{15}$. Mutant predator strains lacking secreted peptidoglycan GlcNAc deacetylases $(\Delta \mathrm{Bd} 0468$ and $\Delta \mathrm{Bd} 3279)$ complete predation but leave behind a "ghost" cell of undigested prey cell-wall material ${ }^{15}$. Peptidoglycan isolated from $E$. coli bdelloplasts is $\mathrm{N}$-deacetylated ( $60-70 \%$ of MurNAc and GlcNAc) and thereby resistant to digestion by conventional lysozymes ${ }^{10}$. Hence, $B$. bacteriovorus and related predators have the potential to encode a deacetylation-specific lysozyme.

Here, we discover a family of Bdellovibrio proteins that define a new subgrouping of the lysozyme superfamily and demonstrate that they possess outright specificity for deacetylated peptidoglycan. We solve the structure of family member Bd0314 (that we named DslA), revealing adaptations to the lysozyme fold that sterically restrict the active-site cleft into only accepting polymers deacetylated at the GlcNAc positions. We determine a role for DslA in B. bacteriovorus exit from the prey cell and demonstrate that exogenously added enzyme is able to prematurely liberate predators from prey bdelloplasts before completion of the predatory lifecycle.

\section{Results}

Lysozyme-like gene bd0314 is expressed during prey exit phase. In the genome of B. bacteriovorus, four divergent/cryptic lysozyme homologs are encoded, Bd0314 (DslA), Bd1413 (DslB), Bd1440 (DslC), and a more distal member Bd1411 (22\% identity to DslA, in comparison to DslB and DslC which are $30 \%$ identical to DslA and $60 \%$ identical to each other). Transcriptional analysis revealed that $b d 1411$ was upregulated in the attachment phase (about $15 \mathrm{~min}$ after infection), whereas bd1413 and bd1440 transcription was not differentially regulated at specific timepoints of the predatory lifecycle of B. bacteriovorus, but expressed throughout (Supplementary Fig. 1). In contrast, bd0314 was upregulated in the late stages (180-240 min after infection) of the predatory lifecycle - around prey exit (Fig. 1). Therefore, Bd0314 (DlsA) was hypothesized to be involved in the exit and release of new progeny $B$. bacteriovorus cells from the inner periplasm of prey bdelloplasts. Further analysis indicated that bd0314 was transcribed alone and not with flanking genes (Supplementary Fig. 2).

The roles of each of the different lysozymes in the predatory lifecycle were addressed by gene deletion $(\Delta b d 0314, \Delta b d 1411$, $\Delta b d 1440)$ or inactivation of an active-site residue (E151Q in Bd1413, resulting in bd1413EQmc). The deletion of bd0314 resulted in a significant delay in prey exit time and is studied further as described below. In comparison, time-lapse microscopy of the entry phase revealed a significantly increased entry time for a mutant deleted for the early-expressed (more distantly related) $b d 1411$, while $\Delta b d 0314$ also indicated a small potential increase ( $p$-value 0.0388 ) in entry time (Supplementary Fig. 3). However, the inactivation of the active-site in Bd1413 or deletion of Bd1440 did not delay the entry process. These data indicate a primary role for Bd1411 in prey entry (which is not studied further in this paper) and an important role for Bd0314 in prey exit.

Bd0314 is important in prey exit. Epifluorescence time-lapse microscopy revealed a delay in the overall exit time of $B$. bacteriovorus $\Delta b d 0314$ in comparison to the wild-type HD100 strain (Fig. 2). The median exit time of the wild type was $65 \mathrm{~min}$, whereas it took $\Delta b d 0314$ a median of $100 \mathrm{~min}$ from predator progeny septation in the bdelloplast until the first progeny cell escaped the prey. Complementation of $\Delta b d 0314$ by the single crossover of pK18-bd0314 with the wild type bd0314 significantly reduced exit time relative to the mutant strain. Complementation did not completely restore the exit phenotype back to wild-type level. This is likely a result of the presence of two bd0314 promoter regions (one in front of the wild-type bd0314 and one in 
a

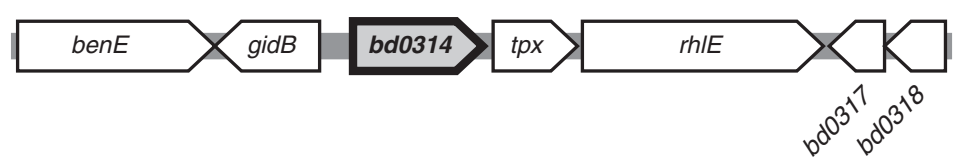

b

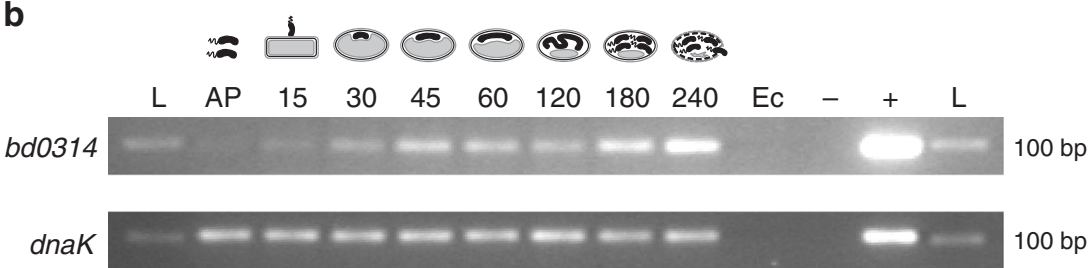

Fig. 1 Transcriptional expression and genomic context of lysozyme homolog-encoding bd0314. a Scheme of the genomic context of bd0314 including surrounding genes predicted to encode a benzoate membrane transport protein (benE), a glucose-inhibited division protein B (gidB), a thiol peroxidase (tpx), an ATP-dependent RNA helicase ( $h / E$ ), and two hypothetical proteins (bd0317, bd0318). b Transcriptional analysis by reverse transcriptase PCR showing the expression of lysozyme gene homolog bd0314 and housekeeping gene dnaK, during the host-dependent lifecycle of $B$. bacteriovorus HD100 as depicted in drawings above the agarose gel. AP: attack phase HD100 cells, 15-240: timepoint in minutes after the invasion, Ec: E. coli S17-1 prey cells, -: negative control with $\mathrm{H}_{2} \mathrm{O}$, +: positive control with genomic DNA of B. bacteriovorous HD100, L: 100 bp DNA ladder. This figure shows one of two biological repeats with similar results.

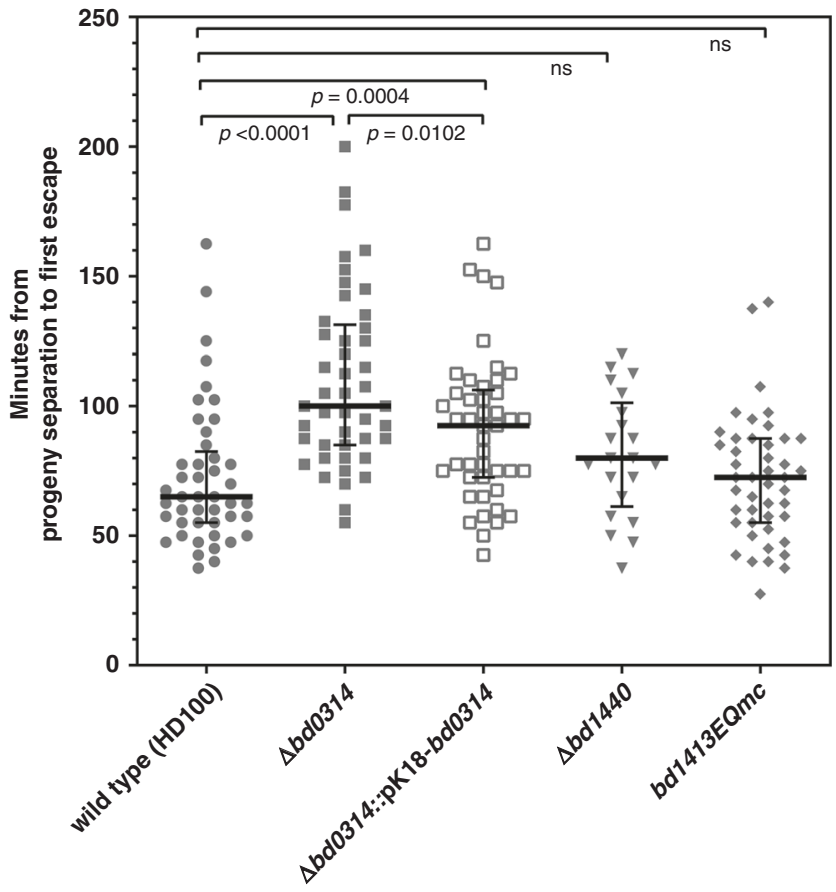

Fig. $2 \Delta b d 0314$ shows a delayed prey exit phenotype. Comparison of time required from progeny separation to the first exit of $B$. bacteriovorus HD100 wild type vs. Dsl- family mutant strains ( $\Delta$ bd0314, $\Delta b d 1440$, and bd1413EQmc) and complemented strain ( $\Delta$ bd0314::pK18-bd0314) from prey cell bdelloplast remnants monitored by epifluorescence time-lapse microscopy. Bdelloplasts were placed on a $1 \%$ agarose slide $\sim 3 \mathrm{~h}$ post-infection of $E$. coli S17-1::pMALp2_mCherry (generating fluorescent prey periplasm and cytoplasm) and observed for another $4 \mathrm{~h} 40$ min to determine backlit predator progeny septation and any exit ${ }^{34}$. Median values and interquartile range are shown as black lines. Data originate from at least two independent biological replicates ( $n=45$ for each strain, except for $\Delta b d 1440$, where $n=21)$. The two-tailed $p$ values are derived from the Mann-Whitney test (ns non-significant, $p$-values are presented in the figure).

front of the deletion remnant of $b d 0314$ ), in the complementation strain, which may have reduced levels of $b d 0314$ gene expression relative to wild-type HD100 strain. Predatory strains with a mutation/deletion of $b d 1413(d s l B)$ or $b d 1440(d s l C)$ did not show a statistically significant increase in exit time compared to wild type. As median exit times of these strains are between the ones of wild type and $\Delta b d 0314(80 \mathrm{~min}$ for $\Delta b d 1440$ and $72.5 \mathrm{~min}$ for $b d 1413 E Q m c)$, a minor role for DslB and DslC in prey exit may be implied. The progeny cells of $\Delta b d 0314$ are trapped for longer within the bdelloplast at the end of predation, which confirms a major role for Bd0314 in the exit process. This implies that the DslA enzyme may act upon the prey peptidoglycan, wherein the GlcNAc residues had been previously deacetylated at the prey invasion stage by B. bacteriovorus Bd3279 and Bd0468 enzymes ${ }^{15}$. For double GlcNAc-deacetylase B. bacteriovorus mutant $\Delta b d 0468$ $\Delta b d 3279$ prey cell-sized "ghost" remnants were abundant in cultures after predation ${ }^{15}$. These large ghost remnants were not seen for the wild type and $\Delta b d 0314$ mutant strain, testing with wheat-germ-agglutinin-Alexa-Fluor-350 conjugate staining (Supplementary Figs. 4 and 5).

DslA structure determination: Exit-specific lysozyme Bd0314 was named DslA for deacetylation-specific lysozyme based on its specific activity as shown later in this article and to distinguish it from the 'original' lysozymes unable to act on deacetylated peptidoglycan ${ }^{6}$. We were able to successfully purify the enzymatic domain of DslA by omitting the lipobox and $\sim 35$ aa region of low complexity/disorder, fusing region R74-K254 to maltose-binding protein (later cleaved). This construct yielded two independent crystal forms, diffracting to 1.26 and $1.36 \AA$ resolution (Table 1). Attempts to solve these via molecular replacement with "conventional" lysozyme structures failed, indicating potential divergence from the superfamily fold.

Large crystals of a catalytic E154Q mutant enabled us to solve the structure via Sulfur-SAD phasing, finding two disulfides and two methionine residues, tracing residues 74-254 inclusive (Fig. 3). The fold is comprised of seven major a-helices (A-G), several smaller $3_{10}$ helices, an elongated $\beta$-hairpin (b1-2) and a smaller $\beta$-hairpin (b3-4). The fold is stabilized by disulfide bonds between C117:C253 and C182:C199. The secondary structure elements create a classical two-lobed architecture, with helix E and $\beta 1-2$ sat below a pronounced active-site cleft (measuring 25 $\AA ̊$ across, $\sim 15 \AA$ deep). The lysozyme superfamily has a conserved ES motif at the $C$ terminus of a central $\alpha$-helix, which is readily identifiable as E143/S144 on DslA helix D (Fig. 3). A second acidic residue, E154 projects upward from the $\beta$-sheet floor of the cleft, and its conformation is stabilized by a hydrogen bond to S166 and packing effects mediated by the hydrophobic sidechains of Y152 and F156. The pose of E154 is further influenced by 
Table. 1 Data collection and refinement statistics.

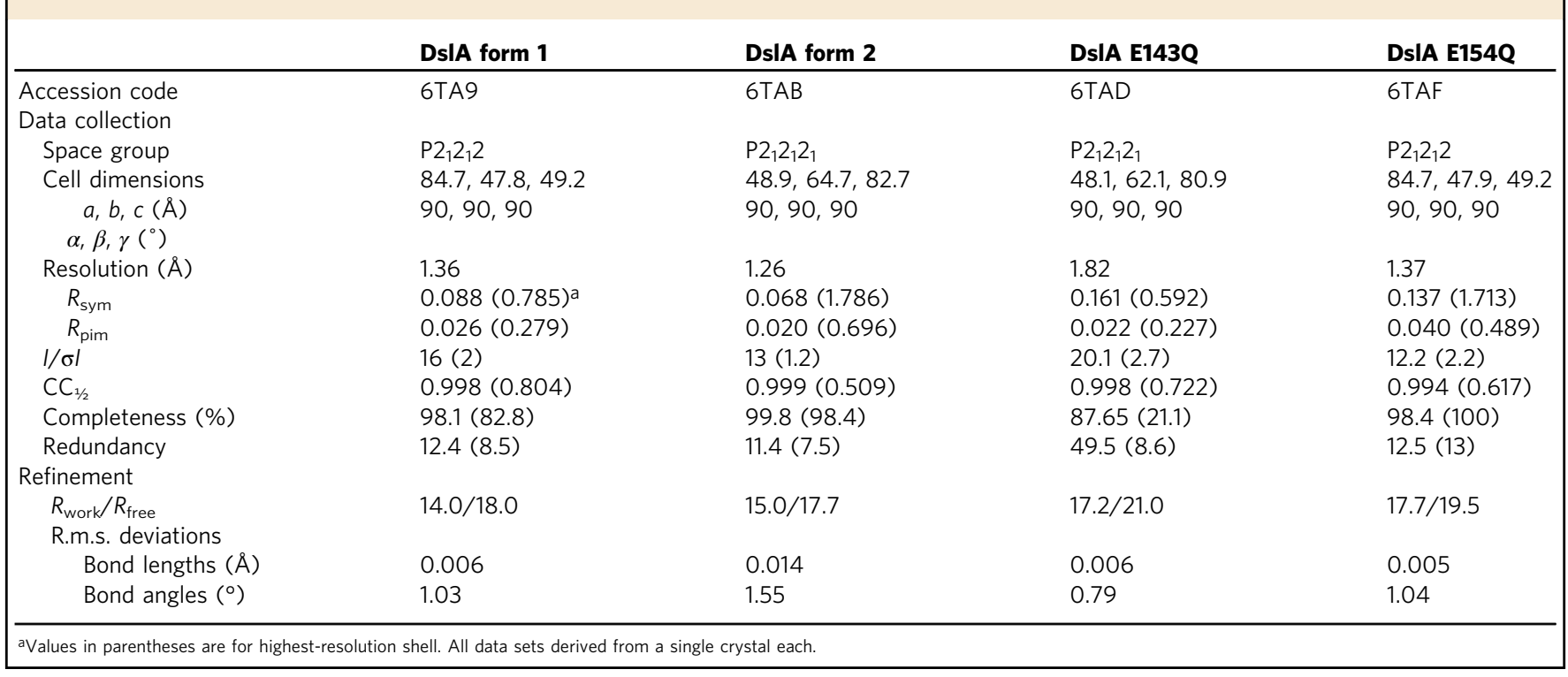

interruption of main chain hydrogen-bonding: the backbone carbonyl groups of E154 and D155 point downward, not participating in $\beta$-hairpin formation. The net effect creates a bulge (Fig. 3c) and allows the sidechain of E154 to face upward, directly across from E143.

Comparison of the DslA fold with structural homologs using DALI $^{16}$ confirmed a partial, but importantly not total, similarity to lysozyme superfamily members. Secondary structure matching confirms that the DslA helix-rich upper lobe is more similar to lytic transglycosylases, e.g., MltF (PDB 4OXV, Z-score 9.8, rmsd $3.5 \AA$, 122 aa alignment at $15 \%$ identity; unpublished but similar to this study) ${ }^{17}$ but this is not significantly higher than a generalized agreement to all lysozymes (e.g., c-type human lysozyme $^{18}, 1 \mathrm{LZS}, 8.3,2.6 \AA$, 104 aa, 13\%). The comparison of all lysozyme superfamily members, including chitinases and chitosanases, noted the conservation of helices D and F, the ES motif and the $\beta$-floor of the cleft ${ }^{19}$; these features are the only factors in agreement when superimposing DslA with the more distantly related GH24 and GH46 enzymes (Fig. 3d). This broader analysis indicates that DslA has four unique regions not shared with any lysozyme subfamily: 73-87 ( $\mathrm{N}$ terminus, helix A), 99-124 (loop region and helix C), $154-164$ (the extended $\beta 1-2$ hairpin past E154), and 214-224 (the $\beta 3-4$ loop), the latter two features extending the depth of the active-site cleft. The tip of the extended $\beta 1-2$ hairpin is stabilized both internally (by the sidechain of D158) and externally (by the sidechain of E175 from the $\mathrm{N}$ terminus of helix E).

Classical lysozyme superfamily catalysis utilizes two acidic residues for hydrolysis or one residue for the GH23/GH102/ GH103/GH104 lytic transglycosylases that generate cyclic 1,6anhydro-MurNAc. The first, as part of the ES motif, is invariable (Fig. 3), but the position of the second alters dependent on retaining versus inverting mechanisms ${ }^{20}$. The second acidic residue of human lysozyme, D53, lies on $\beta 2$ and is equivalent to DslA S166 (Fig. 3e). In contrast, the DslA active-site residue E154 is situated on $\beta 1$ and, despite a relative twisting of the hairpin, is spatially equivalent to the second catalytic residue of v-type lysozymes (e.g., 2LZM D20) and chitosanases (e.g., 4OLT D43) as shown in the zoomed-in comparison of Fig. 3e. In both these other enzyme classes, the second acid is coordinated by a Thr residue, whose equivalent in DslA is S166. The average distance between the DslA E143 and E154 sidechain carboxylates is $8 \AA$, in agreement with the inverting superfamily members that place the second catalytic acid on $\beta 1$ (ref. ${ }^{19}$ ). All four of our DslA structures (two wild-type, E143Q, E154Q) are highly similar, other than a relatively small flexation of the $\beta 1-2$ hairpin in the E154Q variant (Supplementary Fig. 6). The key differences between our structure and conventional lysozymes explain (see the Discussion section) why we went on to find that they have specificity for PG containing deacetylated GlcNAc, unlike conventional lysozymes which are specific for PG containing acetylated GlcNAc.

An enzymatic assay confirms GlcNAc-deacetylase specificity of DslA: We next sought to confirm DslA specificity for GlcNAcdeacetylated peptidoglycan, through use of an enzymatic assay (Fig. 4). This assay uses confirmed B. bacteriovorus GlcNAcdeacetylase proteins ${ }^{15} \mathrm{Bd} 0468$ and $\mathrm{Bd} 3279$ to generate a modified PG substrate from originally acetylated E. coli peptidoglycan labeled with a FITC fluorophore. Hydrolysis was monitored via an increase in fluorescence originating from the conversion of insoluble peptidoglycan to soluble smaller fragments. The assay confirmed the known specificity of hen egg-white lysozyme (HEWL) for acetylated substrate, and lack of specificity for the relatively open active site of mutanolysin ${ }^{6}$. Partial activity for HEWL against the GlcNAc-deacetylase-treated material was indicative of our in vitro prior deacetylation of E. coli PG not running to completion, and illustrative of the difficulties of reconstituting $B$. bacteriovorus activities in vitro outside of the bdelloplast. Despite this, DslA was clearly inactive against native, acetylated peptidoglycan, but displayed strong activity against the deacetylated material; this level of activity was on a similar scale to that of HEWL and mutanolysin with their known substrates.

Using the DslA structure as a guide, we next monitored the activity of DslA mutants for the putative active-site residues. Variants E143Q and E154Q clearly fold and retain a similar active-site architecture (as judged by our high-resolution structures herein, Supplementary Fig. 6), but display no notable activity (Fig. 4, middle), confirming their participation in catalysis. Variant Y228A has residual activity on GlcNAcdeacetylated peptidoglycan but does not convert DslA into a form competent on acetylated material. The presence of Bd1413 (DslB) and Bd1440 (DslC) in B. bacteriovorus HD100 raised the question as to whether they would display similar specificity. We were able to produce these enzymes in a similar manner to DslA, 


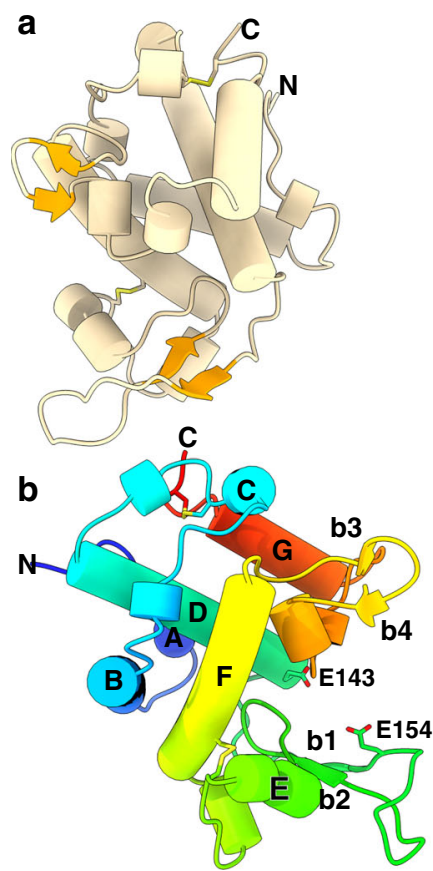

d

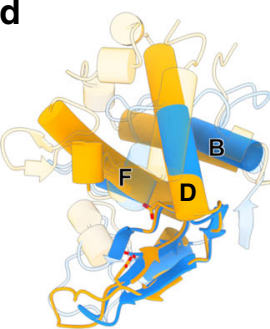

GH22

c-type H. sapiens $1 \mathrm{LZS}$

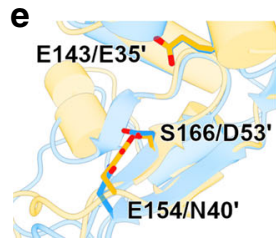

C

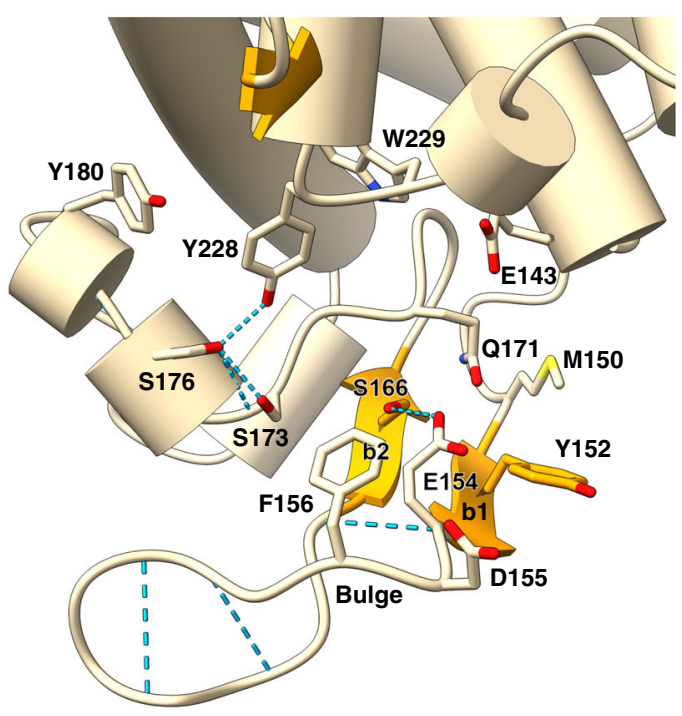

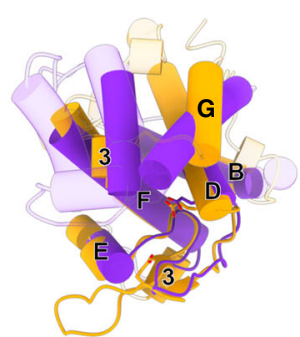

GH23 g-type/lytic TG $P$. aeruginosa 40XV

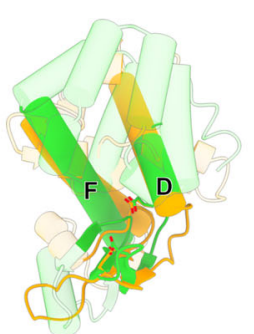

GH24

v-type chitosanase

Phage T4 2LZM Pseudomonas LL2 4OLT

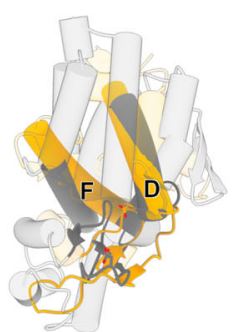

GH46
itosanase

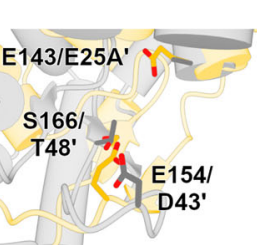

Fig. 3 Structure of DsIA. a Fold of catalytic region of DsIA (R74 to terminal K254), N and C termini labeled, and disulfide bonds presented in stick form. b View $\sim 90^{\circ}$ from a, protein chain colored in rainbow progression from $\mathrm{N}$ terminus (blue) to $\mathrm{C}$ terminus (red), with secondary structure labeled (standard helices via letters A-G; unlabeled helices have been defined as smaller $3_{10}$ helices, $\beta$-strands by numbers b1-4). The two acidic residues identified as participating in catalysis are presented in stick form. c Active site of DsIA, with selected residues in stick form and hydrogen bonds as dashed blue lines. d Comparison between DsIA (orange) and related lysozymes and glycoside hydrolases. The active-site floor $\beta$-sheet and any helices that match are represented in solid form (with DsIA helix designation labeled, lettering includes " 3 " for the smaller $3_{10}$ helices), with non-matching elements rendered transparently. GH numbering refers to CAZy nomenclature 50 , and given examples have organism, PDB code and catalytic residues noted below the fold. Family GH23 demonstrates the strongest similarity to DsIA, with just the core elements (helices $D, F$, $\beta$-sheet) matching for v-type lysozymes and chitosanases. e Zoomed-in active sites from d, with select active-site residues labeled (DsIA numbered, other fold residues noted by a prime ').

and the assay results clearly demonstrate that both DslB and DslC are bone-fide GlcNAc deacetylation-specific lysozymes (Fig. 4, righthand grouping). So although the gene for DslA was most highly expressed at the end of predation, the DslB and C enzymes were also present, possibly in reserve.

Premature release of predator cells from bdelloplasts by exogenous addition of DslA. As DslA showed specific activity toward GlcNAc-deacetylated peptidoglycan in vitro (Fig. 4), we wished to show its activity in vivo. We knew from our previous work $^{15}$ that GlcNAc deacetylases of B. bacteriovorus act on the prey PG during the first $30 \mathrm{~min}$ of predation. To test for DslA activity, invaded E. coli prey bdelloplasts post GlcNAc deacetylation ( $1 \mathrm{~h}$ after predation was started) were used in test treatments with EDTA (to assist enzyme access through the outer membrane) and exogenously added DslA. This $1 \mathrm{~h}$ timepoint is early in the $4 \mathrm{~h}$ predation period when usually no predator cells naturally escape the bdelloplast, but when the GlcNAc of the prey peptidoglycan is already deacetylated. Additional controls were performed using EDTA plus DslA with one of the catalytic site residues inactivated (E143Q), or using conventional hen eggwhite lysozyme (HEWL) or buffer only. An outline of the premature release experiment is shown in Fig. 5a. Images of the treated bdelloplasts (examples shown in Supplementary Fig. 7) 


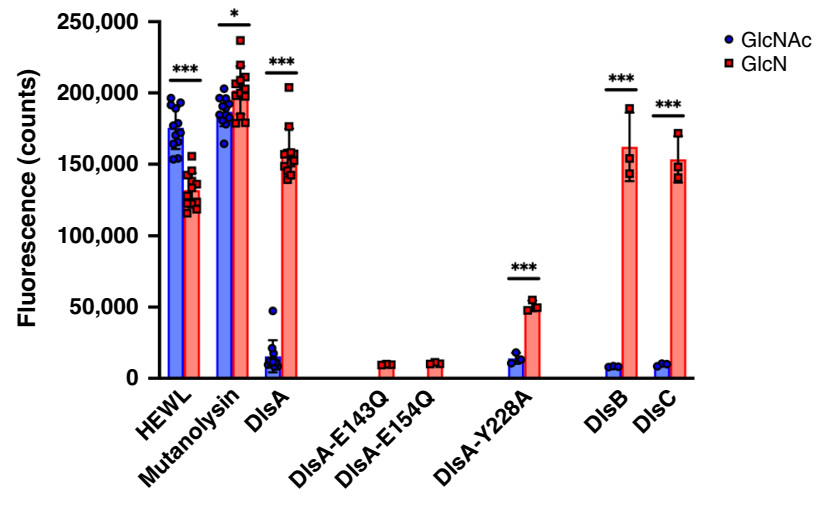

Fig. 4 Enzyme assay of DsIA, related enzymes, and general lysozymes. Enzymes were incubated with acetylated peptidoglycan (GlcNAc, blue) and peptidoglycan partly deacetylated via treatment with Bdellovibrio Bd0468 and $\mathrm{Bd} 3279$ ( $\mathrm{GlcN}$, red). Fluorescence reading monitors the hydrolytic release of FITC-labeled peptidoglycan fragments into solution. Left grouping: comparison of specificity of DslA, hen egg-white lysozyme (HEWL), and Streptomyces globisporus mutanolysin. The non-specific mutanolysin hydrolyzes both acetylated- and deacetylated peptidoglycan equally, but HEWL is specific for the acetylated form (with residual activity on GlcN sample indicative of mixed material, partial $\mathrm{N}$-deacetylation ${ }^{15}$ ). DsIA displays strong activity for the deacetylated GIcN sample and is unable to turnover acetylated GIcNAc peptidoglycan. Middle grouping: relative low activity of DsIA putative catalytic $E$ to $Q$ mutants, and reduced activity for Y228A variant. Right grouping: homologous DsIB and DsIC proteins display a similar preference for a deacetylated substrate. Significance analysis uses two-tailed $t$-test, ${ }^{\star \star \star} p<0.0005,{ }^{\star} p<0.05 . n=12$ independent samples for HEWL, Mutanolysin, and DIsA experiments. $n=3$ independent samples for DIsA mutants and homologs (DIsB and DIsC) experiments. Data are represented as the mean \pm standard deviation.

were categorized manually based on the different detected outcomes (Fig. 5b and Supplementary Fig. 8) and then grouped into superordinate categories dependent on the internal or external position of the Bdellovibrio with respect to the bdelloplast before (Supplementary Fig. 9) and after treatment (Fig. 5c). The percentage of prematurely released, external predator cells increased significantly when early bdelloplasts were treated by exogenous addition of DslA, whereas no significant change was detected for the treatment controls DslA E143Q, HEWL or buffer only (Fig. 5c). This finding was accompanied by a concomitant increase in predatory cells, that appears to be just in the process of being released (Fig. 5c, Bdellovibrio interm.).

Thus, the wild-type DslA, which is normally expressed at the end of predation, could act, when added exogenously with EDTA, on the GlcNAc-deacetylated PG of E. coli prey bdelloplasts and release predators prematurely, as would normally occur naturally later by endogenous predator expression of DslA at the exit stage of predation.

\section{Discussion}

Starting from a theoretical/practical requirement of $B$. bacteriovorus to utilize at least one specialized "exit enzyme", our phenotypic, biochemical and structural experimentation identifies and confirms DslA as a true GlcNAc deacetylation-specific lysozyme with a structure different to other lysozyme superfamily members. In doing so, we have likely identified and structurally defined the primary active agent of the predatory culture concentrate that Ruby and co-authors used in cell extracts, in seminal early work, to prematurely release cells when studying Bdellovibrio differentiation ${ }^{21}$. The usage by B. bacteriovorus of peptidoglycan GlcNAc deacetylation to mark prey versus self is a self-protection mechanism distinct from that previously observed (immunity protein inhibition of effector enzymes ${ }^{13}$ ). Our work here adds to the known "tools" of the remarkable kit of selfprotection which $B$. bacteriovorus developed through evolution to be able to prey upon and destroy bacteria made of very similar chemical components to itself.

Peptidoglycan deacetylation is widely utilized in human and animal host adaptations by Gram-positive bacteria but not typically in Gram-negatives ${ }^{7}$, which may suit its usage by the predator of Gram-negatives $B$. bacteriovorus. However, any prey cell-possessing enzymes acting downstream of deacetylation could potentially autolyze upon invasion or due to exposure to a PG lytic enzyme of self or external predator. Consistent with this idea, it is likely the lipobox of the protein acts to display and retain DslA at the outer leaflet of the predator outer membrane, acting to locally disrupt the prey's bdelloplast wall when predator progeny exit. This would be different if DslA were to be generally secreted on a signal peptide, with the potential for deleterious lysis of neighboring predators yet to complete the intraperiplasmic stage of the lifecycle (or neighboring and as yet uninvaded prey, depending on their PG acetylation status). Thus, the structure and enzymatic mechanism (discussed below), plus the likely tethering of DslA to predator are both important features for efficient predation.

The architecture of DslA is related to, but significantly different from, other members of the lysozyme superfamily (Supplementary Fig. 11) but still fulfil the inclusion criteria for the lysozyme superfamily noted by Monzingo et al. - conservation of the $\beta$ sheet floor and helices $\mathrm{D}$ and $\mathrm{F}$, with appropriate placement of acidic catalytic residues ${ }^{19}$. It is interesting that DslA is structurally more similar to GH23 lytic transglycosylases than GH46 chitosanases - hence, it is probably "easier" for evolution to adapt for sugar selection than muropeptide selection. The structural equivalence of DslA E154 to the $\beta 1$ secondary catalytic acid residues of goose-type lysozymes (also GH23, but with a second catalytic residue lacking in the lytic transglycosylases), v-type lysozymes and chitosanases, and our assay on the E143Q and E154Q mutants argues for an inverting hydrolytic mechanism for DslA. Residue E143 of the conserved ES motif would act as a general acid, protonating the equatorial linkage between MurNAc and GlcN, with E154 acting as a general base to activate water to attack the MurNAc $\mathrm{C} 1$ in an axial orientation ${ }^{19}$. This mechanism is distinct from HEWL and other c-type lysozymes wherein positioning of the second catalytic residue on $\beta 2$ (occupied by Ser166 in DslA, Fig. 3a) results in no space for a water molecule under the substrate and a retaining mechanism ${ }^{22}$.

One of the adaptations of DslA is to utilize a double Glu mechanism - all characterized lysozymes to date use a Glu:Asp pair. The Glu:Glu usage is however conserved in a select group of chitosanases $^{23}$, raising the intriguing possibility that a second Glu could be a favorable feature of deacetylated GlcN-metabolizing enzymes. The assay data confirm a clear specificity of DslA for GlcNAc-deacetylated peptidoglycan - our structure allows us to explain how this is achieved using a variant on the lysozyme superfamily fold. Retention of the ES motif and features surrounding the active-site cleft suggest that it is valid to assume DslA will utilize a similar (invariant) hexasaccharide binding mode to that of other lysozymes. Despite the relatively more extended $\beta$-strand features of DslA, the cleft shape and charge is not significantly different from c-type lysozymes (Fig. 6a). Hence, despite GlcN having a $\mathrm{p} K_{\mathrm{a}}$ of $\sim 6.5$, and thus GlcNAc-deacetylated peptidoglycan possessing a more positive charge than the acetylated form, DslA does not use negatively charged residues to complex the substrate. Using a GH22 chitohexaose complex $(1 \mathrm{LZS})^{18}$ and helix D, and the ES motif as the basis of superimposition, it is possible to model a DslA substrate-binding mode 
a

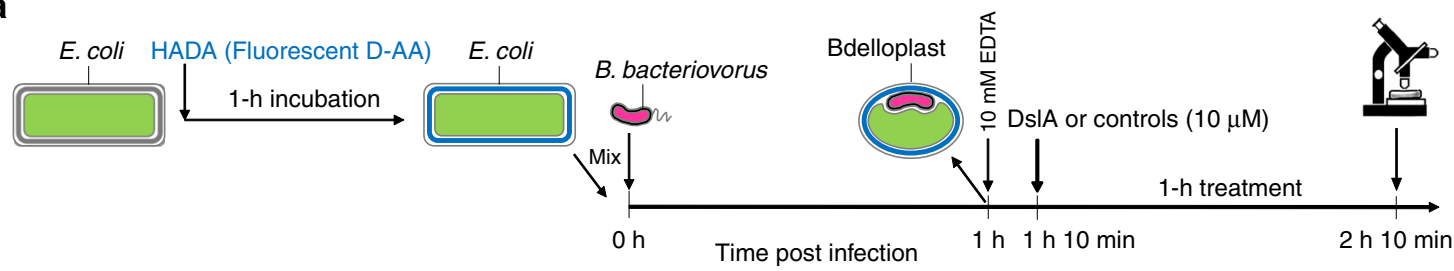

b
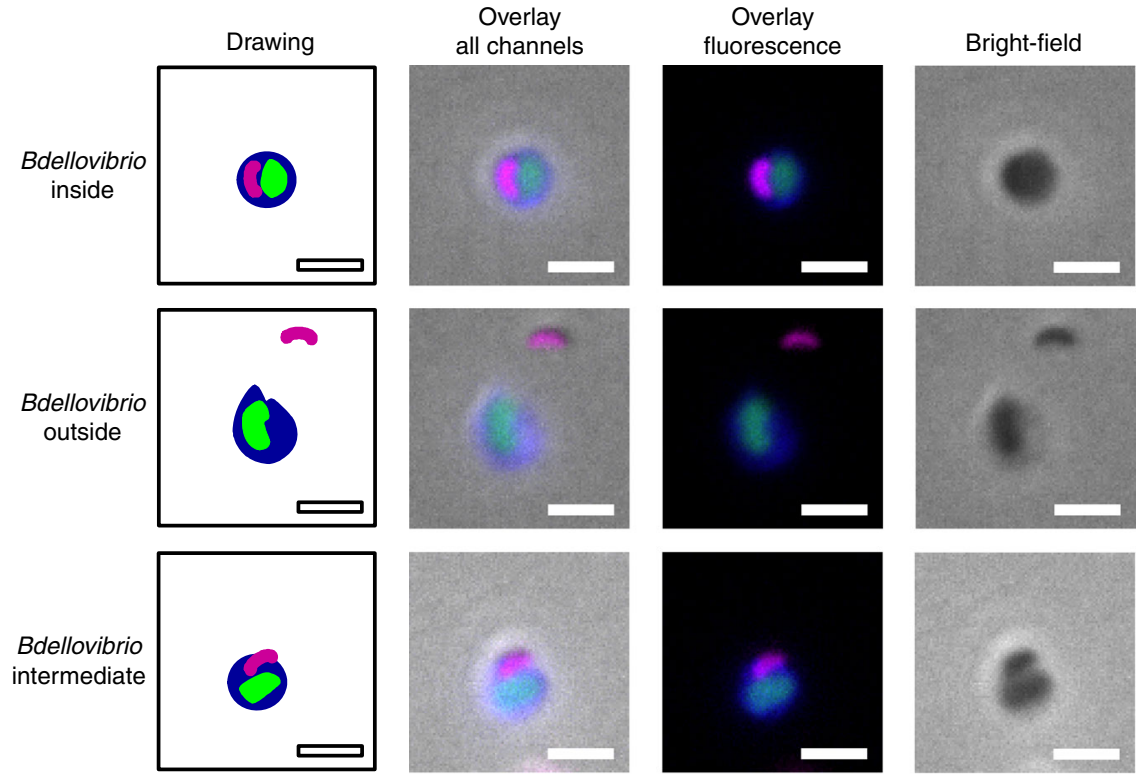

\section{C}
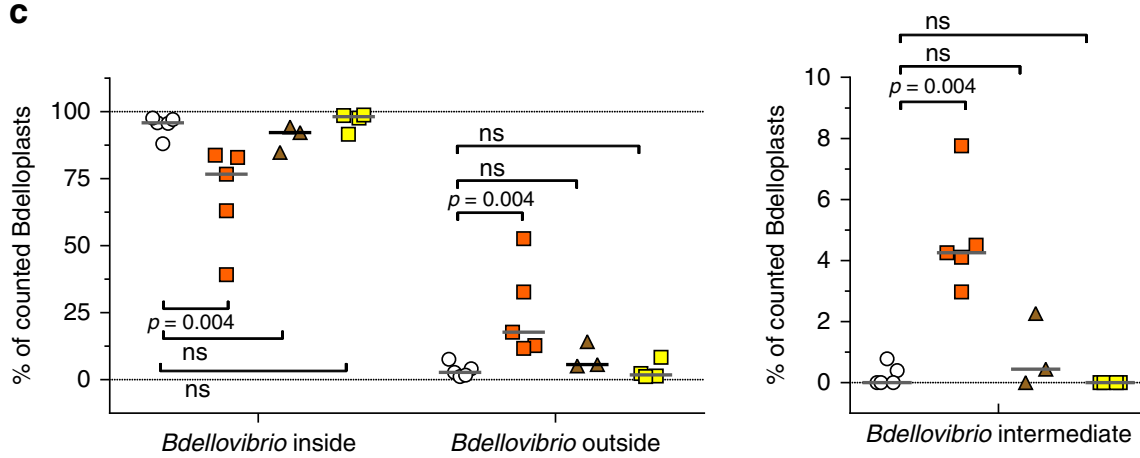

O Buffer control $(n=5)$

$\square$ DsIA wt $(n=5)$

$\triangle \mathrm{DsIAE} 143 \mathrm{Q}(n=3)$

$\square$ HEWL $(n=4)$

Fig. 5 Exogenously added lysozyme DsIA releases B. bacteriovorus from $\mathbf{1} \mathbf{h}$ bdelloplast. a Experimental scheme including different stains/fluorescent proteins to enhance visualization of different compartments. The experimental outline is described in the legend of Supplementary Fig. 8. $\mathbf{b}$ Example images of different outcomes after treatment copied from Supplementary Fig. 8. B. bacteriovorus bd0064:mcherry is shown in magenta, prey peptidoglycan in blue. Scale bar is $2 \mu \mathrm{m}$. c Percentage of bdelloplasts categorized according to the position of the Bdellovibrio in respect to the bdelloplast after treatment as described above (Supplementary Fig. 8). Individual symbols represent \% of all categorized bdelloplasts per experiment. A minimum of 71 bdelloplasts per treatment condition and biological repeat were evaluated (for details please refer to Source Data file). The number of biological repeats is indicated in the legend as $n$ for the respective treatment. Horizontal lines represent median, statistical test was Mann-Whitney, ns non-significant, $p$-values $=0.004$ (all $p$-values are one-tailed). HEWL hen egg-white lysozyme.

(we were unsuccessful with attempts to co-crystallize or soak various glucosamine polymers into all crystal forms). The DslA: substrate model (Fig. 6c, additional complementary models from other substrate complexes shown in Supplementary Fig. 12) are informative on several levels - GlcNAc deacetylation is preferred/utilized over MurNAc deacetylation in the Bd0468:Bd3279: DslA system because the lysozyme fold chiefly recognizes more GlcNAc $2^{\prime}$ units than MurNAc $2^{\prime}$ units, a feature resulting from the bulky $3^{\prime}$ muropeptide substituent of MurNAc requiring placement outside the cleft. Of the six saccharide rings, GlcNAc " $c$ " and " $\mathrm{e}$ " sit with the $2^{\prime}$-NAc groups facing inward. These two positions are occupied in DslA by Y228 and M150, the former placing the sidechain $\mathrm{OH}$ in the same position as an acetyl oxygen. Hence, specificity of DslA for GlcN over GlcNAc presumably arises via steric blockage of ring $\mathrm{c}$ and $\mathrm{e}$ if the sugar groups are acetylated. The positioning and absolute conservation of Y228 appears to be more important than the more variable M150 (Supplementary Fig. 13), and is of interest when comparing to the wider lysozyme superfamily. Residues in the vicinity of M150, including the partly conserved Y152, may assist in collectively creating an environment that favors deacetylated substrate. The greater degree of conservation at Y228 rather than M150 is in agreement with the analysis of the buried-surface area of lysozyme:substrate complexes wherein more contacts are made 
a
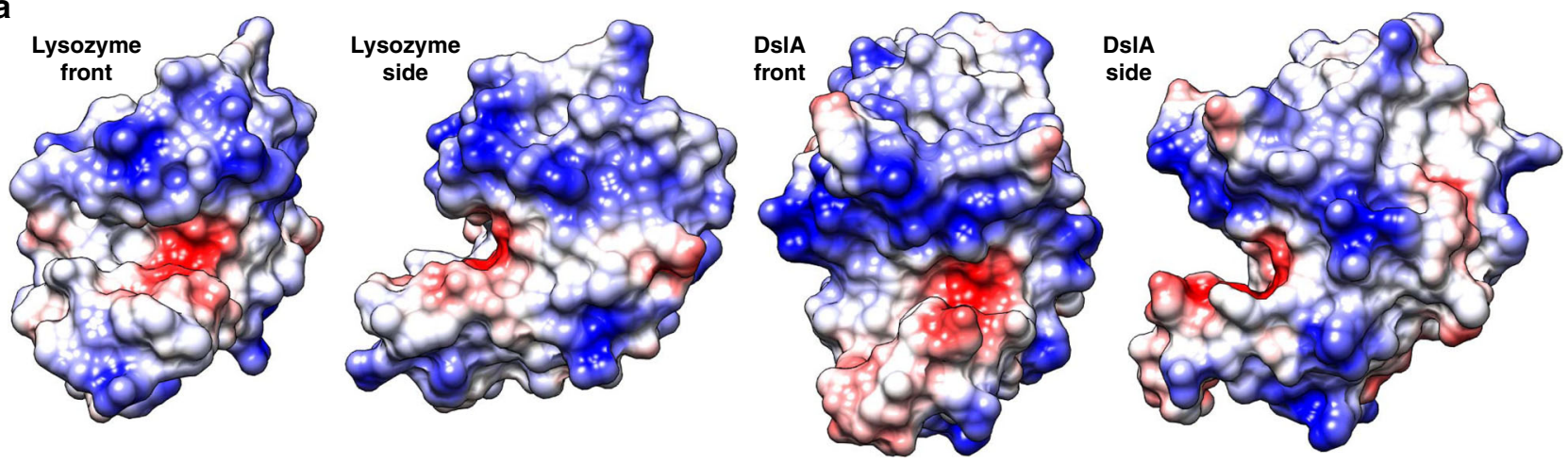

b

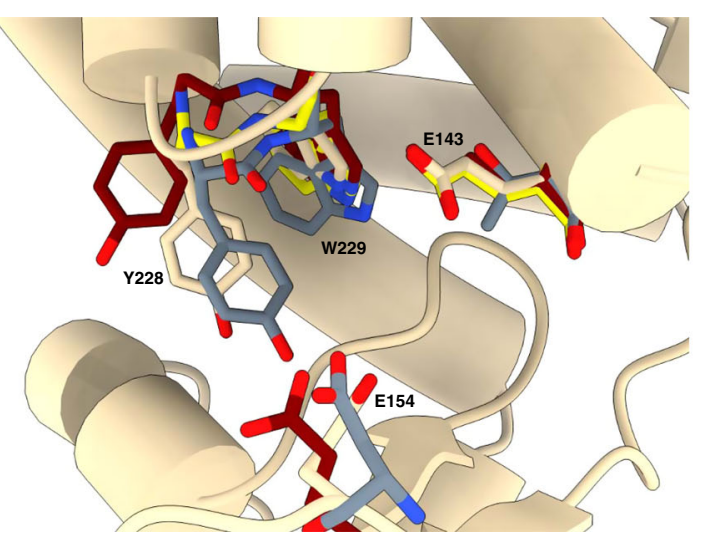

C

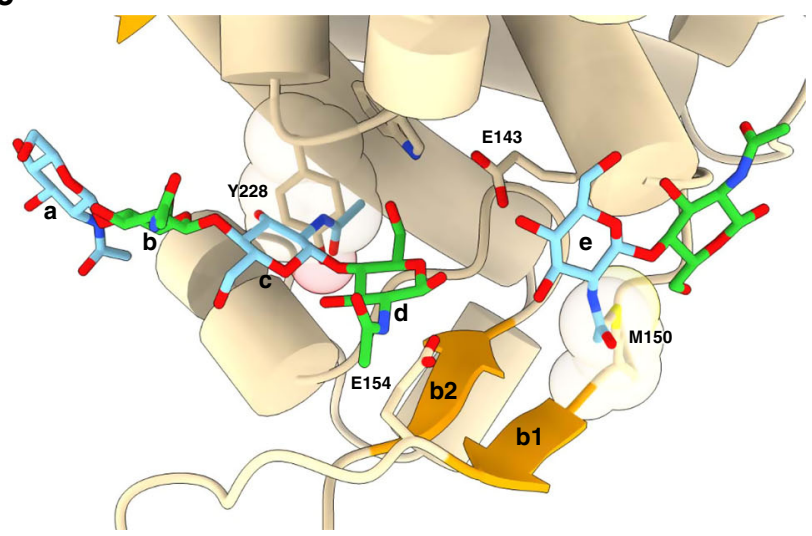

Fig. 6 Active-site cleft and substrate-binding mode. a Surface representation of $H$. sapiens c-type lysozyme (1LZS) and B. bacteriovorus DsIA, with alternating views of active-site cleft. The protein surfaces (1LZS calculated without ligand) have been colored to demonstrate charge using coulombic methods, -10 to $+10 \mathrm{kcal} /(\mathrm{mol} \cdot \mathrm{e})$, red to blue. The two enzymes demonstrate a similar patterning at the active-site cleft, despite DsIA binding deacetylated peptidoglycan. b Comparison of DsIA to non-catalytic members of the lysozyme superfamily. The fold of DsIA and residues E143, E154, and Y228-W229 are shown overlaid with equivalent residues from mouse $\alpha$-lactalbumin (1NF5, gray) ${ }^{24}$, mouse sperm lysozyme-like protein (4YF2, maroon) ${ }^{25}$, and the active enzyme $H$. sapiens lysozyme (1LZS, yellow) ${ }^{18}$. The YW motif on an active-site loop of DsIA is shared with $\alpha$-lactalbumin and sperm lysozyme-like protein and is equivalent to an AW motif in $H$. sapiens lysozyme. The $D$ helix catalytic residue of DsIA, E143, is replaced by Thr in the two inactive enzymes, but the $\beta$-sheet catalytic residue, E154, is retained as either a Glu or an Asp. c DsIA superimposed with substrate from a liganded form of lysozyme (1LZS). The classic "4+2" lysozyme saccharide binding pose is shown, with the ligand coordinates from 1LZS superimposed into DsIA using the ES motif of both enzymes as a guide. The cell-wall sugar rings are labeled a-f, with GlcNAc and MurNAc residues colored blue and green, respectively. The 2' N-acetyl group of rings c and e face inwardly into the rear face of the active-site cleft and provide a model for deacetylation specificity: the sidechains of residues Y228 and M150 (vdw surface shown in transparent) sterically occupy the same space as the acetyl group atoms.

to ring $\mathrm{c}(-2$ position) rather than ring e $(+1)$. Hence, DslA is also likely to follow this pattern, discriminating most strongly via the central region of the active-site cleft.

We were able to find two structurally characterized wider superfamily members that retain a YW motif at the location of DslA Y228:W229; most lysozymes have a smaller residue preceding the tryptophan (e.g., AW in 1LZS, Fig. 6b). Interestingly, these two YW variants, $\alpha$-lactalbumin ${ }^{24}$ and mouse sperm lysozyme-like protein ${ }^{25}$, are both annotated as inactive enzymes. Hence, like DslA, these proteins may use the YW motif to block binding of acetylated GlcNAc peptidoglycan. Mouse sperm lysozyme-like protein and $\alpha$-lactalbumin replace the first catalytic residue equivalent to E143 with a catalytically incompetent Thr, but retain an equivalent to the second acidic residue DslA E154 (Fig. 6b). We observe a lesser activity for the Y228A variant of DslA (Fig. 4), but this does not "convert" to an enzyme competent in turning over acetylated peptidoglycan - thus, substrate selection is likely to involve additional factors to steric block by Y228, possibly selecting for the subtly different torsion angles between GlcNAc and GlcN or other differences in recognition. One of these factors may be the topology of the peptide backbone in this region; the carbonyl of Y228 projects out relatively further than equivalent residues in the superfamily, and mutation to alanine will not nullify this feature. Factors additional to Y228 that could potentially contribute to specificity may be complex and beyond the scope of this study (e.g., active-site dynamics, or enzyme/substrate hydration differences in relation to other superfamily members). Chitosanases often use tyrosine to recognize the amine group of $\mathrm{GlcN}^{26}$, and the local hydrogenbonding network of DslA Y228-S176-S188 looks to be a suitable pocket for recognition of ring c GlcN.

Our biochemical observation of a similar GlcNAc deacetylation-specificity for DslB and DslC (30\% identity to DslA and encoded as a back-up similar to other duplicated predatory enzyme genes of $B$. bacteriovorus as for DacBs ${ }^{12}$ and GlcNAc deacetylases ${ }^{15}$ ) gives confidence to the process of identifying Dsl homologs via sequence comparison. Using the DslA sequence, we were able to find homologs in several other prey-invasive Bdellovibrio strains (60-70\% identity). However, in line with invasive predatory association and replication of $B$. bacteriovorus within the prey cell wall, we did not find homologs in the epibiotic predators that have no requirement for an exit enzyme (a search of the genomes of the model epibiotic predators B. exovorus and Micavibrio aeruginosavorus revealed no DslABC homologs (no 
hits with an $E$-value $<0.1)$ ). A range of sequences from diverse bacteria were obtained that have $24-43 \%$ sequence identity (Supplementary Fig. 13), and can be validated in that all sequences possessing an equivalent of E154 also have absolute conservation of Y228. Several of these strains have identifiable CE4 deacetylase genes, and so will be able to generate the GlcN cell-wall substrate. Hence, it will be interesting to see if these systems use coupled GlcNAc deacetylation and GlcN-hydrolysis in a biologically important manner. An in-depth analysis of the roles of DslB and DslC, which were not differentially expressed at exit time in B. bacteriovorus, is beyond the scope of this study. However, we did show that B. bacteriovorus strains with mutated/ deleted DslB or DslC showed small but not significant delays in prey entry or exit compared to wild type (Fig. 2 and Supplementary Fig. 3), thus they potentially represent a spare, secondary, enzyme set. Gene duplication giving rise to pairs of enzymes contributing to a single PG-modifying task has been previously demonstrated for D,D-endopeptidases ${ }^{12}$ and $N$-deacetylases ${ }^{15}$ in $B$. bacteriovorus. As we found in a previous study that $50 \%$ of GlcNAc-deactylase double deletion mutant strains get "trapped" in the outer periplasm between prey wall and outer membrane ${ }^{15}$, there may be a requirement for a localized Dsl-activity during entry of predators to prey, as prey wall deacetylation is occurring at the time of invasion. (Other enzymes are also involved in the multi-factorial invasion process ${ }^{14}$ and are the subject of a current extensive experimental program beyond this study.) The majority of the lysozyme-like activity on prey entry seems to be from Bd1411, with a potential minor contribution by DslA (Supplementary Fig. 3). Although predominant gene expression (Fig. 1) and DslA activity is in the exit phase (Fig. 2), it is possible that some DslA enzyme originating from the exit remains attached to the Bdellovibrio outer membrane, as it is a lipoprotein. In this way DslA could persist after exit and potentially still be used at entry, which will be the subject of another multienzyme study.

The fact that the $\Delta d s l A$ mutant did ultimately leave the prey cells after a delay (Fig. 2) indicates that additional predatory enzymes are involved in the overall exit process which does involve breakage of the whole outer envelope, including wall but also an outer membrane of the prey bdelloplast. There was no obvious difference in the way by which $\Delta d s l A$ mutant predator progeny break the prey cell into remnants on exit in comparison to the wild-type exit mode (Supplementary Fig. 14). This contrasts the two different exit modes observed for the double GlcNAc-deacetylase mutant where some whole prey cell-sized "ghost" remnants were seen in cultures after predation ${ }^{15}$. This can be explained by the different timepoints respective enzymes are active within the predatory lifecycle and the outcomes of them being missing in B. bacteriovorus mutants. GlcNAc-deacetylase enzymes were previously found to act early in predation at invasion time and their deletion in a double $\Delta b d 0468$ bd3279 B. bacteriovorus mutant caused approximately half of the predators to fail to enter through the prey cell wall, instead carrying out predation in the outer periplasm, between the wall and outer membrane, and approximately half entering the inner periplasm. The outer dwelling half of the $\Delta b d 0468$ bd3279 deacetylase mutant population left behind intact prey cell "ghosts" of wall and membranes (seen by fluorescently labeled prey cytoplasm being detected in osmotically stable spheres) after predation. The inner dwelling half of the population exited through the original pore by which they had traversed the prey cell wall - again leaving behind smaller prey remnants. In contrast, the $\Delta d s l A$ mutant has a different phenotype (Supplementary Fig. 15) and the population predominantly cross the prey wall, likely due to the $\Delta d s l A$ mutant still expressing active Bd1411, and grow in the inner periplasm (expression of $d s l A$ being higher after prey invasion), without leaving extensive prey remnants.

Our work here identifies DslA as important to B. bacteriovorus predatory biology, and confirms that evolution has shaped a divergent lysozyme homolog family to be specific for GlcNAcdeacetylated peptidoglycan, and that, importantly, this enzyme contributes to prey cell exit by predatory bacteria. The duplication and diversification of known cell wall-metabolizing activities for a "new" purpose in the predatory B. bacteriovorus lifecycle is a theme observed before for D,D-endopeptidases ${ }^{12}$ and L,D-transpeptidases $^{14}$, and can be inferred for other activities also ${ }^{27}$. DslA (or homologs), if adapted for Gram-positive peptidoglycan may find use as a tool in the study of such pathogens that deacetylate their wall to escape conventional lysozyme activity ${ }^{4}$. Our observations will assist in unraveling the complexity of Bdellovibrio prey cell manipulation and provide a basis for identifying the biological role of cryptic homologs of DslA.

\begin{abstract}
Methods
Growth of bacterial strains. Bdellovibrio bacteriovorus strain $\mathrm{HD} 100^{\mathrm{T}}$ was used throughout, and wild type, or mutated $\Delta b d 0314(\Delta d s l A), \Delta b d 0314:: \mathrm{pK} 18-b d 0314$, $\Delta b d 1411, \Delta b d 1440(\Delta d s l C), b d 1413:: p K 18-b d 1413 E Q m C h e r r y(b d 1413 E Q m c)$, or bd0064:mcherry strains were maintained on Ca/HEPES buffer ( $25 \mathrm{mM}$ HEPES, 2 $\mathrm{mM} \mathrm{CaCl}_{2}, \mathrm{pH}$ 7.6) with late-log phase E. coli S17-1 prey (if not indicated differently) at $29^{\circ} \mathrm{C}$ for $16-24 \mathrm{~h}$ with $200 \mathrm{rpm}$ shaking ${ }^{28-30}$. Various $E$. coli strains were grown in YT broth at $37^{\circ} \mathrm{C}$ and $200 \mathrm{rpm}$ plus additional substances as indicated, if required.
\end{abstract}

RNA isolation from predatory lifecycle/HI grown cells and RT-PCR analysis Synchronous predatory infections of B. bacteriovorus HD100 on prey E. coli S17-1 were set up by concentrating 11 predatory cultures by centrifugation at $16,900 \times g$ and resuspending in $100 \mathrm{ml} \mathrm{Ca} / \mathrm{HEPES}$ buffer, then mixing $50 \mathrm{ml}$ of this with $30 \mathrm{ml}$ $\mathrm{Ca} / \mathrm{HEPES}$ buffer and $40 \mathrm{ml}$ E. coli $\mathrm{S} 17-1$ back diluted to $\mathrm{OD}_{600} 1.0 \mathrm{in} \mathrm{Ca} / \mathrm{HEPES}$ buffer $^{31}$. Controls of predator only and prey only were also set up. HI stains were grown up to an $\mathrm{OD}_{600 \mathrm{~nm}}$ of 0.6 and all samples were treated as with $1 \%$ phenol, $19 \%$ ethanol for $45 \mathrm{~min}$ at $4{ }^{\circ} \mathrm{C}$, before centrifugation at $5000 \times g$ for $10 \mathrm{~min}$ and the pellet stored at $-80^{\circ} \mathrm{C}$ (ref. ${ }^{31}$ ). RNA was isolated from these pellets using the Promega SV total RNA isolation kit with RNA quality being verified by an Agilent Bioanalyser using the RNA Nano kit. All samples for Supplementary Fig. 2 with the

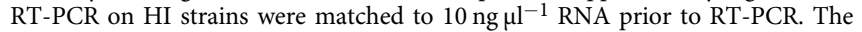
Qiagen One-step RT-PCR kit was used to perform the following reaction conditions: one cycle $50{ }^{\circ} \mathrm{C}$ for $30 \mathrm{~min}, 94^{\circ} \mathrm{C}$ for $15 \mathrm{~min}$, then $25-30$ amplification cycles of $94^{\circ} \mathrm{C}$ for $1 \mathrm{~min}, 50^{\circ} \mathrm{C}$ for $1 \mathrm{~min}, 72^{\circ} \mathrm{C}$ for $1 \mathrm{~min}$, and finally a $10 \mathrm{~min}$ extension at $72^{\circ} \mathrm{C}$ after the last cycle, followed by a $4{ }^{\circ} \mathrm{C}$ hold. All experiments to monitor transcription during $\mathrm{HD}$ lifecycle were carried out with at least two biological repeats. RT-PCR primers to amplify an $\sim 100$-bp-long fragment were $5^{\prime}$-TATCGA ACCGGAAGTCGTTC- $3^{\prime}$ and $5^{\prime}$-GCAACCTTGTCTTCCCAAAG- $3^{\prime}$ for $b d 0314$ (dslA), $5^{\prime}$-ACAAACAGAAGGACCGCAAG- $3^{\prime}$ and $5^{\prime}$-TCATCACATGACGACCC AAC- $3^{\prime}$ for $b d 1411,5^{\prime}$-CTCCAGAGCCTGAACCAGAG- $3^{\prime}$ and $5^{\prime}$-CCTGCAGG TATTTGGACCAC- $3^{\prime}$ for $b d 1413(d s l B), 5^{\prime}$-TGGGAAACTTCCACAAATCC- $3^{\prime}$ and $5^{\prime}$-GGCAGAACTTGCTCATGTCA- $3^{\prime}$ for $b d 1440(d s l C)$, and $5^{\prime}$-TGAGGAC GAGATCAAACGTG- $3^{\prime}$ and $5^{\prime}$-AAACCAGGTTGTCGAGGTTG- $3^{\prime}$ for $d n a K$ (bd1298).

Generation and complementation of lysozyme mutants in $B$. bacteriovorus HD100. Markerless deletion mutants $\Delta b d 0314(\Delta d s l A)$ and $\Delta b d 1411$ of the $b d 0314$ and $b d 1411$ open reading frames in B. bacteriovorus HD100 were generated using a modified version of Steyert and Pineiro ${ }^{32}$ by subcloning $1 \mathrm{~kb}$ of up- and downstream flanking DNA first in pUC19, then excising this fragment and cloning into the suicide vector pK18mobsacB ${ }^{33}$, which was then transformed into E. coli S17-1 for conjugating into wild-type $B$. bacteriovorus HD100. Conjugations were carried out for $16 \mathrm{~h}$ at $29^{\circ} \mathrm{C}$ on PY agar $\left(10 \mathrm{~g} \mathrm{l}^{-1}\right.$ Difco Bacto peptone, $3 \mathrm{~g} \mathrm{l}^{-1}$ Difco Yeast extract $10 \mathrm{~g} \mathrm{l}^{-1}$ agar) by concentrating $10 \mathrm{ml}$ of a predatory B. bacteriovorus and 10 $\mathrm{ml}$ donor $E$. coli S17-1 by centrifugation at $5500 \times \mathrm{g}$ for $10 \mathrm{~min}$ and resuspending each in $100 \mu \mathrm{l} \mathrm{Ca} / \mathrm{HEPES}$ buffer and placing on a nylon membrane. Exconjugants were then grown as predatory cultures in Ca/HEPES buffer supplemented initially with $50 \mu \mathrm{g} \mathrm{ml}^{-1}$ kanamycin selection, then subsequently in Ca/HEPES buffer supplemented with $5 \%$ sucrose to select for a second crossover ${ }^{29,30}$. The primers to generate the deletion mutant were: Bd0314-F 5'-AGCTACGCATGCACCGCCAG AACCAAAATCCG-3'; Bd0314- $\triangle$ R 5'-GCTGGTCTTGTCACCGGCACTCTAGA CCAGGATCTGAACAACAAGC-3'; Bd0314-R 5'-CTTAGCGGATCCGGCGAT GATATCAGAACCAT-3'; Bd0314- $\Delta \mathrm{F}$ 5'-GCTTGTTGTTCAGATCCTGGTCTAG AGTGCCGGTGACAAGACCAGC-3'; Bd1411-F 5'-TGCTAGAAGCTTGTTGTG AATCACTCATTGCC-3'; Bd1411- $\Delta$ R 5'-CGCCTGATCTTACTTGCAGCATGC AGCAAGCATGATGCCGGA-3'; Bd1411-R 5'-GACTAGTCTAGATCTCTGGTG 
AGATCAACACG-3'; and Bd1411- $\Delta \mathrm{F}$ 5'-TCCGGCATCATGCTTGCTGCATGCT GCAAGTAAGATCAGGCG-3'

Markerless deletion mutant $\Delta b d 1440(\Delta d s l C)$ was generated by assembling 220 bp upstream region and first $6 \mathrm{bp}$ of open reading frame $b d 1440$ with the last $9 \mathrm{bp}$ of the reading frame with 299 bp downstream flanking DNA. For Gibson assembly with the NEBuilder kit (New England Biolabs) the following primers where used: Bd1440-F 5'-CGTTGTAAAACGACGGCCAGTGCCAATCCCCGGCCATCAGC CA-3'; Bd1440- $\Delta$ R 5'-GTTACTTACACGTCAAAAGACCTCCAAAGTGTC-3'; Bd1440- $\Delta$ F 5' -TCTTTTGACGTGTAAGTAACAAGACGGTCGGG-3', and Bd14 40-R 5'-GGAAACAGCTATGACCATGATTACGGTCTTCAAAGTTGTCCCG-3'. Complementation of $\Delta b d 0314$ was achieved by single crossover with plasmid $\mathrm{pK} 18$ bd0314 containing a kanamycin resistance. Plasmid pK18-bd0314 was conjugated into B. bacteriovorus $\Delta b d 0314$ from donor E. coli strain S17-1 as described above. Plasmid pK18-bd0314 containing the $b d 0314$ (wild type) open reading frame with 1000-bp upstream and 61-bp downstream region on conjugable vector $\mathrm{pK} 18 \mathrm{mobsacB}{ }^{33}$ was generated by Gibson assembly with the NEBuilder kit (New England Biolabs) with primers Bd0314Comp-F 5'-CGTTGTAAAACGACGGCCAGTGCCAATCGCCTT GTTTGCGACAATTG-3' and Bd0314Comp-R 5'-GGAAACAGCTATGACCATGA TTACGGGATGCTCCTTTTGTTTAAGCG-3' using gDNA of $B$. bacteriovorus HD100 as a template. Strain bd1413::pK18-bd1413EQmCherry (bd1413EQmc) was obtained by a single crossover with plasmid pK18-bd1413EQmCherry containing a kanamycin resistance. Plasmid pK18-bd1413EQmCherry was conjugated into $B$. bacteriovorus HD100 wild type from donor E. coli strain S17-1 as described above. Plasmid pK18-bd1413EQmCherry for the inactivation of the active-site E151 (to $\mathrm{Q} 151$ ) of Bd1413 (DslB) in was achieved by mutagenic PCR using the primers Bd1413mCherryF 5'-GTACTGGAATTCATGATCGCAACAAAGAACCA-3', 1413 FOE 5'-CGCCAAATATCAGAGCGCCTACAG-3', Bd1413mCherryR 5'-TGACGA GGTACCTTTGCACAAGGGAAGAGTCT- ${ }^{\prime}$ and 1413ROE $5^{\prime}$-CTGTAGGCGCTC TGATATTTGGCG-3'. The resulting PCR product was subcloned into pAKF56 (named pMAL_p2-mCherry in Fenton et al. ${ }^{34}$ ) with EcoRI and KpnI to fuse $m$ Cherry with the mutated $b d 1413$. This was then cloned into the conjugable vector pK18mobsacB with EcoRI and HindIII.

\section{Time-lapse microscopy to examine entry time. Phase-contrast time-lapse} microscopy was carried out on predation of E. coli S17-1 prey by B. bacteriovorus HD100 wild type, $\Delta b d 0314, \Delta b d 1411$, and $\Delta b d 1440$. B. bacteriovorus strain bd1413EQmc was tested by predation on E. coli S17-1:pZMR100 (ref. ${ }^{31}$ ) in Ca/ HEPES buffer with kanamycin. Fifty times concentrated $24 \mathrm{~h}$ incubated predatory Bdellovibrio culture was mixed with an equal volume of $5 \times$ concentrated late-log phase E. coli prey by centrifugation $(17,000 \times g, 2 \mathrm{~min}$, room temperature). Immediately after mixing, $10 \mu \mathrm{l}$ of the mixture was applied onto a $0.3 \%$ agarose in $\mathrm{Ca} /$ HEPES buffer pad on a microscope slide for phase-contrast time-lapse microscopy. Imaging was performed at ambient temperature with the Nikon Eclipse E600 microscope using a $\times 100$ objective lens (numerical aperture (NA), 1.25), and an exposure time of $0.01 \mathrm{~s}$ (gain 0). Images were acquired using a Hamamatsu Orca ER camera and Simple PCI software (version 5.5). Sequential imaging every $1 \mathrm{~min}$ for six fields of view was enabled by an H101A $x y$ motorized stage (Prior Scientific, minimum step size, $0.01 \mu \mathrm{m}$ ), and a frictional $z$ axis controller (minimum step size, $2 \mathrm{~nm}$ ) in conjunction with the Simple PCI software, which enabled fine autofocusing. Measurements are from at least three independent biological replicates with 90 events evaluated per predatory strain.

Time-lapse microscopy to determine exit time. 24-h incubated cultures of $B$. bacteriovorus HD100 wild type, $\Delta b d 0314$ or $\Delta b d 1440$ growing predatorily on a late$\log$ phase E. coli S17-1 culture in Ca/HEPES buffer at $29^{\circ} \mathrm{C}$ and $200 \mathrm{rpm}$ were filtered through a $0.45 \mu \mathrm{m}$ filter and concentrated $50 \times$ by centrifugation $(3877 \times g$ for $20 \mathrm{~min}$ at $29^{\circ} \mathrm{C}$ ). The same procedure was performed with $B$. bacteriovorus bd1413::pK18-bd1413EQmCherry (bd1413EQmc) or $\Delta b d 0314:$ :pK18-bd0314 pregrown on $E$. coli S17-1:pZMR100 (ref. ${ }^{31}$ ) prey in Ca/HEPES buffer with $50 \mu \mathrm{g} \mathrm{ml}$ ${ }^{-1}$ kanamycin. However, to minimize kanamycin carry over Bdellovibrio cells were washed once with $\mathrm{Ca} / \mathrm{HEPES}$ buffer containing no antibiotics (additional 5-min spin at $3877 \times g$ and $29^{\circ} \mathrm{C}$ ). Each culture of B. bacteriovorus was mixed with an equal amount of $E$. coli S17-1::pMAL-p2_mCherry ${ }^{34}$ (grown overnight in YT with $50 \mu \mathrm{g} \mathrm{ml}^{-1}$ ampicillin and $200 \mu \mathrm{g} \mathrm{ml}^{-1}$ IPTG at $37^{\circ} \mathrm{C}$ and $200 \mathrm{rpm}$, concentrated to $\mathrm{OD}_{600 \mathrm{~nm}} 5.0$ and washed twice in $\mathrm{Ca} / \mathrm{HEPES}$ buffer). This semi-synchronous infection was incubated at $29^{\circ} \mathrm{C}$ and $200 \mathrm{rpm}$ for $2 \mathrm{~h} 45 \mathrm{~min}$ before a $10 \mu \mathrm{l} \mathrm{sample}$ was transferred onto a $1 \%$ agarose in Ca/HEPES buffer pad on a microscope slide for time-lapse microscopy. Time-lapse epifluorescence microscopy was performed using the same Nikon Eclipse E600 microscope system as described for the entry time-lapse microscopy. Exposure time was $0.1 \mathrm{~s}$ for bright field (gain 0 ) and $4.1 \mathrm{~ms}$ (gain 255) for hcRED filter block, and eight fields of view were imaged sequentially every $2.5 \mathrm{~min}$ per experiment. Measurement of prey exit times - defined as the time between predator progeny separation (estimated frame in which the predator spiral cellular filament structure divides to form separate progeny) and first escape from prey cell remnants (first frame in which a predator progeny cell visibly starts to leave the prey structure and subsequently moves out by at least half a progeny cell length) - was from at least two biological repeats per strain. Only bdelloplasts were evaluated, which were reasonably in focus, fluorescence was bright enough and B. bacteriovorus progeny cells had started to exit up to $280 \mathrm{~min}$ after starting time-lapse microscopy ( $n=45$ for each strain, except for $\Delta b d 1440$, where $n=21$ ). All Serial images were visually inspected using Fiji/ImageJ $(1.52 \mathrm{i})^{35,36}$.

Determining the number of prey residual structures per $\boldsymbol{E}$. coli. Cultures of $B$ bacteriovorus $\mathrm{HD} 100$ wild type or $\Delta b d 0314$ were grown on late-log phase E. coli $\mathrm{K}$ $12 \mathrm{MG} 1655$ in $\mathrm{Ca} / \mathrm{HEPES}$ buffer at $29^{\circ} \mathrm{C}$ and $200 \mathrm{rpm}$ for $24 \mathrm{~h}$. These predatory cultures were filtered $(0.45 \mu \mathrm{m})$, concentrated $10 \times$ by centrifugation $(3877 \times g$ for $20 \mathrm{~min}$ at $29^{\circ} \mathrm{C}$ ) and then incubated at least $10 \mathrm{~min}$ at $29^{\circ} \mathrm{C}$ and $200 \mathrm{rpm}$ before infection of prey. An overnight prey culture of E. coli K-12 MG1655 (grown in YT at $37^{\circ} \mathrm{C}$ and $200 \mathrm{rpm}$ ) was filtered through an autoclaved Millex ${ }^{\circ}$-AP filter (Prefilter 25MM, Millex-AP glass fiber membrane, Merck) to remove any aggregated/ elongated prey cells. The filtered $E$. coli K-12 MG1655 culture was diluted to an $\mathrm{OD}_{600 \mathrm{~nm}} 1.0$ with $\mathrm{Ca} / \mathrm{HEPES}$ buffer, spun for $2 \mathrm{~min}$ at $17,000 \times g$ and resuspended in the same volume of $\mathrm{Ca} / \mathrm{HEPES}$ buffer. A semi-synchronous predatory infection was set up by mixing $0.3 \mathrm{ml}$ Ca/HEPES buffer with $0.4 \mathrm{ml} E$. coli K-12 MG1655 $(\mathrm{OD}=1.0)$ and $0.5 \mathrm{ml} \mathrm{B.} \mathrm{bacteriovorus} \mathrm{HD} 100$ wild type or $\Delta b d 0314(10 \times$ concentrated), and incubated at $29^{\circ} \mathrm{C}$ and $200 \mathrm{rpm}$. Prey remnants were stained $5 \mathrm{~h}$ post-infection with Film tracer $^{\mathrm{TM}} \mathrm{FM}^{\mathrm{TM}} 1-43$ green biofilm cell stain used to stain lipids (final concentration $5 \mu \mathrm{g} \mathrm{ml}^{-1}$, ThermoFisher Scientific) and peptidoglycan stained with Wheat Germ Agglutinin (WGA)-Alexa Fluor 350 conjugate (final concentration $10 \mu \mathrm{g} \mathrm{ml}^{-1}$, ThermoFisher Scientific). After stain addition, samples were incubated for $15 \mathrm{~min}$ in the dark. For determination of the number of bdelloplasts, samples were spiked with latex beads (polystyrene, $0.3 \mu \mathrm{m}$ mean particle size, Sigma) at $1 \mathrm{~h}$ and $5 \mathrm{~h}$ post-infection. Samples were imaged using a Nikon Ti-E inverted fluorescence microscope equipped with Plan Apo $\lambda 100 \times / 1.45$ Ph3 objective, an Andor Neo sCMOS camera, a FITC filter cube and NIS-Elements AR software (version 4.6). Samples were viewed and imaged in phase contrast, in the DAPI channel (Ex: $395 \mathrm{~nm} / \mathrm{Em}$ : $435 \mathrm{~nm}$ ) to detect the WGA-Alexa Fluor 350 conjugate-stained prey peptidoglycan remnants and in the GFP channel (Ex: $470 \mathrm{~nm} / \mathrm{Em}: 515 \mathrm{~nm}$ ) to view membranes stained with FM 1-43. Exposure time was $142 \mathrm{~ms}$ for phase contrast (at gain 1, 12-bit), $2 \mathrm{~s}$ for DAPI channel and $70 \mathrm{~ms}$ for GFP channel (both acquisitions at gain 4, 12-bit). Ten images per strain (wild type or $\Delta b d 0314)$ and timepoint (1- or 5-h post-infection) were processed using Fiji/ImageJ software (1.52i) $)^{35,36}$. All images were cropped to a size of $110.03 \mu \mathrm{m} \times$ $45.30 \mu \mathrm{m}$ visually selecting for the parts most in focus. These images were used for bead, bdelloplast and prey cell remnant counting. Detection and counting of prey cell remnants was performed on the fluorescence images obtained in the DAPI channel in Fiji PlugIn MicrobeJ (version 5.11z) ${ }^{37}$. Latex beads and bdelloplasts (for the 1-h post-infection samples only) were counted manually using the Cell Counter function in Fiji/Image ${ }^{35,36}$. The ratio of bdelloplasts (rounded prey cells infected with Bdellovibrio) to latex beads at $1 \mathrm{~h}$ post-infection was calculated and compared to the ratio of WGA-Alexa Fluor 350 conjugate-stained prey remnants versus polystyrene beads at $5 \mathrm{~h}$ post-infection. Four independent biological repeats were evaluated.

Cloning, expression, and purification. Constructs of $d s l A$ (bd0314, AA 74-254), $d s l B$ (bd1413, AA 80-271), and $d s l C$ (bd1440, AA 75-266), all lacking signal peptides, lipobox, and region of presumed disorder, were amplified from $\mathrm{B}$. bacteriovorus HD100 genomic DNA. The amplified genes were inserted into a modified pET28b-MBP expression plasmid (TEV-cleavable His $_{6}$-Maltose-Binding Protein tag) by restriction-free cloning. Mutant variants of DslA were produced by standard site-directed mutagenesis. Constructs were confirmed by sequencing, and introduced into the commercially available E. coli SHuffle T7 expression strain (NEB) in order to promote disulfide bond formation. LB media supplemented with $1 \% \mathrm{w} / \mathrm{v}$ glucose was used to culture expression strains, and protein production induced at $\mathrm{OD}_{600}$ of 0.6 using $1 \mathrm{mM}$ IPTG (temperature lowered to $18^{\circ} \mathrm{C}$, cells were grown overnight)

Cell pellets were resuspended with appropriate amounts of lysis buffer $(50 \mathrm{mM}$ HEPES pH 7.5, $20 \mathrm{mM}$ imidazole, $300 \mathrm{mM} \mathrm{NaCl}, 0.05 \% \mathrm{w} / \mathrm{v}$ Tween 20) and lysed using sonication, with centrifugation at $33,000 \times g\left(4{ }^{\circ} \mathrm{C}\right)$ for $1 \mathrm{~h}$ to clarify the lysate. The supernatant was then loaded onto HisTrap HP columns (GE Healthcare) preequilibrated in lysis buffer. Columns were washed with $\sim 20$ column volumes of lysis buffer and protein eluted using elution buffer (50 mM HEPES pH 7.5, $300 \mathrm{mM}$ $\mathrm{NaCl}, 400 \mathrm{mM}$ imidazole). TEV protease was added (1:50 ratio) to pooled elution fractions and the sample dialyzed overnight at $4{ }^{\circ} \mathrm{C}$ (in $20 \mathrm{mM}$ HEPES pH 7.5, $200 \mathrm{mM} \mathrm{NaCl}$ ). The cleaved protein was separated from residual fusion protein by a second passage over the HisTrap column. The resulting purified protein was concentrated to $\sim 15 \mathrm{mg} \mathrm{ml}^{-1}$ and utilized in crystallization and kinetics experiments.

Structure determination. Crystals were grown at $18^{\circ} \mathrm{C}$ using the sitting drop technique with drops composed of equal volumes of protein and reservoir solution. For wild-type DslA, crystals were obtained in $0.1 \mathrm{M} \mathrm{Na}$ acetate $\mathrm{pH} 5.5$ and $50 \% \mathrm{w} / \mathrm{v}$ polyethylene glycol (PEG) 400 (P2 $22_{1} 2$ form), or $0.2 \mathrm{M}$ Lithium sulfate, $0.1 \mathrm{M} \mathrm{Na}$ acetate $\mathrm{pH} 4.5$, and $30 \%$ w/v PEG 8000 (P2 $2_{1} 2_{1} 2_{1}$ form). For DslA E143Q and E154Q mutants, crystals were obtained in $0.2 \mathrm{M}$ Lithium sulfate, $0.1 \mathrm{M} \mathrm{Na}$ citrate $\mathrm{pH} 5.5$, and $20 \% \mathrm{w} / \mathrm{v}$ PEG 3000 . Other than the $\mathrm{P} 2{ }_{1} 2{ }_{1} 2$ form (flash cooled directly), crystals were cryoprotected in mother liquor supplemented with $20 \%$ (v/v) ethylene glycol before being flash cooled in liquid nitrogen. Diffraction data were collected at the Diamond 
Light Source in Oxford, UK. Data reduction and processing were completed using $\mathrm{XDS}$ and the $x i a 2$ suit $^{38}$. Initial phasing of DslA was achieved using a merged sulfurSAD data set (9000 frames, $0.1^{\circ}$ oscillations, using two distinct regions of the crystal to minimize radiation damage) of an E143Q crystal collected at a long wavelength of $1.77 \AA$ A . Unmerged data were input into PHENIX AutoSol ${ }^{39}$, which located $13 \mathrm{~S}$ sites with a FOM of 0.3 , allowing iterative cycles of building and model-based phasing improvement. The other crystal forms were solved using the E143Q search model in PHASER $^{40}$. Protein structures were built/modified using COOT $^{41}$, with cycles of refinement in PHENIX ${ }^{39}$ and PDB-REDO ${ }^{42}$.

Peptidoglycan digestion assay. Isolated peptidoglycan (E. coli) was labeled with FITC in a similar method to that described by Maeda et al. ${ }^{43}$. Briefly, $\sim 28 \mathrm{mg}$ of peptidoglycan was incubated with $8 \mathrm{mg}$ of FITC in $300 \mu \mathrm{l} \mathrm{H}_{2} \mathrm{O}$ for $4 \mathrm{~h}$, before being washed and resuspended in deacetylation/assay buffer $(20 \mathrm{mM}$ HEPES pH 7.5, 300 $\mathrm{mM} \mathrm{NaCl}$ ). For deacetylation-specific assays, labeled peptidoglycan was incubated at $37^{\circ} \mathrm{C}$ for $12 \mathrm{~h}$ with two Bdellovibrio deacetylases, Bd0468 and Bd $3279(1 \mu \mathrm{M}$ each). All assayed enzymes (DslABC, HEWL, mutanolysin) were incubated in assay buffer, with the 60 min time course using $100 \mu \mathrm{g} \mathrm{ml}^{-1}$ peptidoglycan and $5 \mathrm{ng}$ of enzyme, and samples taken at regular intervals, with the reaction quenched by removing the insoluble substrate using a $0.1 \mu \mathrm{m}$ filter. Fluorescence counts were measured using a PheraStar FS plate reader set to excite at $485 \mathrm{~nm}$ and detect at $520 \mathrm{~nm}$.

Early bdelloplast-treatment assay. A schematic overview of this experiment is given in Fig. 5a. B. bacteriovorus bd0064:mcherry (B. bacteriovorus with a fluorescent cytoplasm $)^{44}$ instead of the wild-type strain was used to clearly distinguish between predatory cells and other components present in the assay at the end of treatment with DslA or controls. B. bacteriovorus bd0064:mcherry was grown for $24 \mathrm{~h}$ on latelog-phase E. coli S17-1 prey in Ca/HEPES buffer. This prey lysate culture was filtered $(0.45 \mu \mathrm{m})$ and concentrated $20 \times$ by centrifugation $\left(20 \mathrm{~min}\right.$ at $\left.3877 \times \mathrm{g}, 29^{\circ} \mathrm{C}\right)$. The protocol of Kuru et al. ${ }^{14}$ was used as guidance to stain the prey PG with HADA. An overnight prey culture of E. coli S17-1 pAKF220 (ref. ${ }^{45}$ ) (expressing mNeonGreen in the cytoplasm), grown induced with $200 \mu \mathrm{g} \mathrm{ml}^{-1}$ IPTG on YT with $50 \mu \mathrm{g} \mathrm{ml}^{-1}$ ampicillin, was diluted to an $\mathrm{OD}_{600 \mathrm{~nm}}$ of 1.0 with YT. After pelleting the cells ( 2 min at $17,000 \times g$ at room temperature), the cells were resuspended in an equal amount of YT (without ampicillin) and $500 \mu \mathrm{M} \mathrm{HADA}^{46,47}$ (a fluorescent D-amino acid). This mixture was incubated for $30 \mathrm{~min}$ at $37^{\circ} \mathrm{C}$ and $200 \mathrm{rpm}$. Labeled E. coli S17-1 pAKF220 was washed twice ( $2 \mathrm{~min}$ at $17,000 \times \mathrm{g}$ at room temperature) in a volume corresponding to $\mathrm{OD}_{600 \mathrm{~nm}} 1.0 \mathrm{in} \mathrm{Ca} / \mathrm{HEPES}$ buffer. The infection was started by mixing equal volumes of concentrated B. bacteriovorus bd0064:mcherry with labeled E. coli S17-1 pAKF220 and subsequent incubation at $29^{\circ} \mathrm{C}$ and $200 \mathrm{rpm}$. Semisynchronicity of infection was confirmed by microscopic observation at $30 \mathrm{~min}$ postinfection. At one hour post infection $10 \mathrm{mM}$ ethylenediaminetetraacetic acid (EDTA) was added to the lysate containing early bdelloplasts and the mixture was incubated for $10 \mathrm{~min}$ (at $29^{\circ} \mathrm{C}, 200 \mathrm{rpm}$ ) to mediate access of the exogenously added enzymes. DslA, DslA E143Q, and hen egg-white lysozyme (Sigma) were added to the assay at a final concentration of $10 \mu \mathrm{M}$, whereas an equal volume of Ca/HEPES buffer was added for the buffer control. These treatment assays were incubated for one hour at $29^{\circ} \mathrm{C}$ and $200 \mathrm{rpm}$. Just after the addition or $1 \mathrm{~h}$ of treatment with DslA or controls $5 \mu \mathrm{l}$ from the assay was transferred onto a $1 \%$ agarose in Ca/HEPES buffer pad on a microscope slide. Samples were imaged using a Nikon Ti-E inverted fluorescence microscope equipped with Plan Apo $\lambda \times 100 / 1.45$ Ph3 objective, an Andor Neo sCMOS camera, a FITC filter cube and NIS-Elements AR (version 4.6). Samples were imaged in phase contrast (exposure $4.8 \mathrm{~ms}$ ), the DAPI channel (Ex: $395 \mathrm{~nm} / \mathrm{Em}$ : $435 \mathrm{~nm}$, exposure $100 \mathrm{~ms}$ ) the GFP channel (Ex: $470 \mathrm{~nm} / \mathrm{Em}$ : $515 \mathrm{~nm}$, exposure $20 \mathrm{~ms}$ ) and the mCherry channel (Ex: $555 \mathrm{~nm} / \mathrm{Em}$ : $632 \mathrm{~nm}$, exposure $1 \mathrm{~s}$ ) (with all acquisitions at gain 4 ). Fiij/ImageJ software (1.52i $)^{35,36}$ was used for image processing. Bdelloplasts that were reasonably in focus were sorted manually by visual inspection into the different categories shown in Supplementary Fig. 8. An overview of the categorized bdelloplasts including total numbers are given in the Source Data xls spreadsheet. The percentage of each category with respect to all bdelloplasts counted under the given conditions were calculated and merged into superordinate categories based on the position of Bdellovibrio with respect to the PG of the bdelloplast (please refer to Source Data). The different numbers of independent biological repeats for different treatments evaluated are indicated in Fig. 5 and Supplementary Fig. 9 as $n$.

Phylogenetic tree. Protein sequences were aligned using ClustalW and phylogenetic trees were generated in MEGA-X software version 10.0.05, using the Maximum Likelihood method with 500 bootstraps ${ }^{48}$. Trees were visualized in FigTree version 1.4.4 (http://tree.bio.ed.ac.uk/software/figtree/). Additional protein homologs were found through the NCBI BLAST program suite ${ }^{49}$.

Reporting summary. Further information on research design is available in the Nature Research Reporting Summary linked to this article.

\section{Data availability}

Coordinates and structure factors have been deposited in the PDB under accession codes $6 \mathrm{TA} 9$ and $6 \mathrm{TAB}$ for the two wild-type protein crystal forms; 6TAD for the E143Q mutant; and $6 \mathrm{TAF}$ for the E154Q mutant. Source data are provided with this paper for
Figs. 1b, 2, 4, 5c and Supplementary Figs. 1b, 2a, S3, 5, and 9 in the Source Data file. Other data are available from the corresponding author upon request. Source data are provided with this paper.

Received: 30 October 2019; Accepted: 6 August 2020; Published online: 23 September 2020

\section{References}

1. Fleming, A. \& Allison, V. D. Observations on a bacteriolytic substance ("Lysozyme") found in secretions and tissues. Br. J. Exp. Pathol. 3, 252-260 (1922).

2. Blake, C. C. F. et al. Structure of hen egg-white lysozyme: a three-dimensional fourier synthesis at $2 \AA$ resolution. Nature 4986, 757-761 (1965).

3. Callewaert, L. \& Michiels, C. W. Lysozymes in the animal kingdom. J. Biosci. 35, 127-160 (2010).

4. Araki, Y., Fukuoka, S., Oba, S. \& Ito, E. Enzymatic deacetylation of Nacetylglucosamine residues in peptidoglycan from Bacillus cereus cell walls. Biochem. Biophys. Res. Commun. 45, 751-758 (1971).

5. Dong, T. G., Ho, B. T., Yoder-Himes, D. R. \& Mekalanos, J. J. Identification of T6SS-dependent effector and immunity proteins by Tn-seq in Vibrio cholerae. Proc. Natl Acad. Sci. USA 110, 2623-2628 (2013).

6. Vollmer, W., Joris, B., Charlier, P. \& Foster, S. Bacterial peptidoglycan (murein) hydrolases. FEMS Microbiol. Rev. 32, 259-286 (2008).

7. Vollmer, W. Structural variation in the glycan strands of bacterial peptidoglycan. FEMS Microbiol. Rev. 32, 287-306 (2008).

8. Sockett, R. E. Predatory lifestyle of Bdellovibrio bacteriovorus. Annu. Rev. Microbiol. 63, 523-539 (2009).

9. Thomashow, M. F. \& Rittenberg, S. C. Intraperiplasmic growth of Bdellovibrio bacteriovorus 109J: solubilization of Escherichia coli peptidoglycan. J. Bacteriol. 135, 998-1007 (1978).

10. Thomashow, M. F. \& Rittenberg, S. C. Intraperiplasmic growth of Bdellovibrio bacteriovorus 109J: N-deacetylation of Escherichia coli peptidoglycan amino sugars. J. Bacteriol. 135, 1008-1014 (1978).

11. Thomashow, M. F. \& Rittenberg, S. C. Intraperiplasmic growth of Bdellovibrio bacteriovorus 109J: attachment of long-chain fatty acids to escherichia coli peptidoglycan. J. Bacteriol. 135, 1015-1023 (1978)

12. Lerner, T. R. et al. Specialized peptidoglycan hydrolases sculpt the intrabacterial niche of predatory Bdellovibrio and increase population fitness. PLoS Pathog. 8, e1002524 (2012)

13. Lambert, C. et al. Ankyrin-mediated self-protection during cell invasion by the bacterial predator Bdellovibrio bacteriovorus. Nat. Commun. 6, 8884 (2015).

14. Kuru, E. et al. Fluorescent D-amino-acids reveal bi-cellular cell wall modifications important for Bdellovibrio bacteriovorus predation. Nat. Microbiol. 2, 1648-1657 (2017).

15. Lambert, C. et al. Interrupting peptidoglycan deacetylation during Bdellovibrio predator-prey interaction prevents ultimate destruction of prey wall, liberating bacterial-ghosts. Sci. Rep. 6, 26010 (2016).

16. Holm, L. \& Laakso, L. M. Dali server update. Nucleic Acids Res. 44, W351-W355 (2016)

17. Domínguez-Gil, T. et al. Activation by allostery in cell-wall remodeling by a modular membrane-bound lytic transglycosylase from Pseudomonas aeruginosa. Structure 24, 1729-1741 (2016).

18. Song, H., Inaka, K., Maenaka, K. \& Matsushima, M. Structural changes of active site cleft and different saccharide binding modes in human lysozyme co-crystallized with hexa-N-acetyl-chitohexaose at pH 4.0. J. Mol. Biol. 244, 522-540 (1994).

19. Monzingo, A. F., Marcotte, E. M., Hart, P. J. \& Robertus, J. D. Chitinases, chitosanases, and lysozymes can be divided into procaryotic and eucaryotic families sharing a conserved core. Nat. Struct. Biol. 3, 133-140 (1996).

20. Mhlongo, N. N., Skelton, A. A., Kruger, G., Soliman, M. E. \& Williams, I. H. A critical survey of average distances between catalytic carboxyl groups in glycoside hydrolases. Proteins 82, 1747-1755 (2014).

21. Ruby, E. G. \& Rittenberg, S. C. Differentiation after premature release of intraperiplasmically growing Bdellovibrio bacteriovorous. J. Bacteriol. 154, 32-40 (1983).

22. Vocadlo, D. J., Davies, G. J., Laine, R. \& Withers, S. G. Catalysis by hen egg white lysozyme proceeds via a covalent intermediate. Nature 412, 835-838 (2001).

23. Yorinaga, Y., Kumasaka, T., Yamamoto, M., Hamada, K. \& Kawamukai, M. Crystal structure of a family 80 chitosanase from Mitsuaria chitosanitabida. FEBS Lett. 591, 540-547 (2017).

24. Ramakrishnan, B. \& Qasba, P. K. Crystal structure of lactose synthase reveals a large conformational change in its catalytic component, the beta1,4galactosyltransferase-I. J. Mol. Biol. 310, 205-218 (2001). 
25. Zheng, H. et al. Sperm Lysozyme-Like Protein 1 (SLLP1), an intra-acrosomal oolemmal-binding sperm protein, reveals filamentous organization in protein crystal form. Andrology 3, 756-771 (2015).

26. Saito, J. et al. Crystal structure of chitosanase from Bacillus circulans MH-K1 at 1.6-A resolution and its substrate recognition mechanism. J. Biol. Chem. 274, 30818-30825 (1999).

27. Lambert, C., Chang, C. Y., Capeness, M. J. \& Sockett, R. E. The first bite-profiling the predatosome in the bacterial pathogen Bdellovibrio. PLoS ONE 5, e8599 (2010).

28. Lambert, C. \& Sockett, R. E. Laboratory maintanance of Bdellovibrio. Curr. Protoc. Microbiol. 9, 7B.2.1-7B.2.13 (2008).

29. Capeness, M. J. et al. Activity of Bdellovibrio hit locus proteins, Bd0108 and Bd0109, links type IVa pilus extrusion/retraction status to prey-independent growth signalling. PLoS ONE 8, e79759 (2013).

30. Lambert, C. \& Sockett, R. E. Nucleases in Bdellovibrio bacteriovorus contribute towards efficient self-biofilm formation and eradication of preformed prey biofilms. FEMS Microbiol. Lett. 340, 109-116 (2013).

31. Lambert, C. et al. Characterizing the flagellar filament and the role of motility in bacterial prey-penetration by Bdellovibrio bacteriovorus. Mol. Microbiol. 60, 274-286 (2006).

32. Steyert, S. R. \& Pineiro, S. A. Development of a novel genetic system to create markerless deletion mutants of Bdellovibrio bacteriovorus. Appl. Environ. Microbiol. 73, 4717-4724 (2007)

33. Schäfer, A. et al. Small mobilizable multi-purpose cloning vectors derived from the Escherichia coli plasmids pK18 and pK19: selection of defined deletions in the chromosome of Corynebacterium glutamicum. Gene 145, 69-73 (1994)

34. Fenton, A. K., Kanna, M., Woods, R. D., Aizawa, S. I. \& Sockett, R. E. Shadowing the actions of a predator: backlit fluorescent microscopy reveals synchronous nonbinary septation of predatory Bdellovibrio inside prey and exit through discrete bdelloplast pores. J. Bacteriol. 192, 6329-6335 (2010).

35. Rueden, C. T. et al. ImageJ2: ImageJ for the next generation of scientific image data. BMC Bioinformatics 18, 529 (2017).

36. Schindelin, J. et al. Fiji: an open-source platform for biological-image analysis. Nat. Methods 9, 676-682 (2012).

37. Ducret, A., Quardokus, E. M. \& Brun, Y. V. MicrobeJ, a tool for high throughput bacterial cell detection and quantitative analysis. Nat. Microbiol. 1, 16077 (2016).

38. Winter, G., Lobley, C. M. \& Prince, S. M. Decision making in xia2. Acta Crystallogr. D Biol. Crystallogr. 69, 1260-1273 (2013).

39. Zwart, P. H. et al. Automated structure solution with the PHENIX suite Methods Mol. Biol. 426, 419-435 (2008).

40. McCoy, A. J. et al. Phaser crystallographic software. J. Appl. Crystallogr. 40, 658-674 (2007).

41. Emsley, P. \& Cowtan, K. Coot: model-building tools for molecular graphics. Acta Crystallogr. D Biol. Crystallogr. 60, 2126-2132 (2004).

42. Joosten, R. P., Joosten, K., Cohen, S. X., Vriend, G. \& Perrakis, A. Automatic rebuilding and optimization of crystallographic structures in the Protein Data Bank. Bioinformatics 27, 3392-3398 (2011).

43. Maeda, H. A new lysozyme assay based on fluorescence polarization or fluorescence intensity utilizing a fluorescent peptidoglycan substrate. J. Biochem. 88, 1185-1191 (1980).

44. Willis, A. R. et al. Injections of predatory bacteria work alongside host immune cells to treat Shigella Infection in zebrafish larvae. Curr. Biol. 26, 3343-3351 (2016).

45. Makowski, L. et al. Dynamics of chromosome replication and its relationship to predatory attack lifestyles in Bdellovibrio bacteriovorus. Appl. Environ. Microbiol. 85, https://doi.org/10.1128/AEM.00730-19 (2019).

46. Kuru, E. et al. In situ probing of newly synthesized peptidoglycan in live bacteria with fluorescent D-amino acids. Angew. Chem. Int Ed. Engl. 51, 12519-12523 (2012).

47. Kuru, E., Tekkam, S., Hall, E., Brun, Y. V. \& Van Nieuwenhze, M. S. Synthesis of fluorescent D-amino acids and their use for probing peptidoglycan synthesis and bacterial growth in situ. Nat. Protoc. 10, 33-52 (2015).
48. Kumar, S., Stecher, G., Li, M., Knyaz, C. \& Tamura, K. MEGA X: molecular evolutionary genetics analysis across computing platforms. Mol. Biol. Evol. 35, $1547-1549$ (2018)

49. Altschul, S. F., Gish, W., Miller, W., Myers, E. W. \& Lipman, D. J. Basic local alignment search tool. J. Mol. Biol. 215, 403-410 (1990).

50. Lombard, V., Golaconda Ramulu, H., Drula, E., Coutinho, P. M. \& Henrissat, B. The carbohydrate-active enzymes database (CAZy) in 2013. Nucleic Acids Res. 42, D490-D495 (2014).

\section{Acknowledgements}

This work was funded by BBSRC BB/M010325/1 and BB/J015229/1 (to R.E.S. and A.L.L. for C.L. and R.T.), BBSRC DTP studentships (to C.H., L.J.R. and H.S.), SNSF Postdoc Mobility fellowships P2SKP3_174680 and P400PB_183896 (to S.G.H.), and BBSRC BB/ S010122/1 (to P.J.M.). R.E.S. and A.L.L. are currently supported by a Wellcome Trust Investigator Award 209437/Z/17/Z

\section{Author contributions}

All authors contributed to experimental design, which was supervised by A.L.L. for protein biochemistry and structure determination and by R.E.S. for microbiological assays and gene expression; C.H. cloned and purified DslABC, did all the structural characterizations, and assayed enzymes in vitro with assay design and supervision by P.J.M. S.H. conducted and evaluated all the video exit phenotypes of B. bacteriovorus mutants with help of C.L. All mutants (except $\Delta d s l C$ ) were constructed and partially characterized by H.S. and C.L. Entry phenotypes were assayed by H.S. and S.H. with the assistance of C.L. S.H. used DslA protein produced by C.H. to perform ex vivo premature release assays; and carried out microscopy and image analysis with assistance from C.L. G.T. constructed $\Delta d s l C$ and conducted two repeats of RT-PCR on $b d 1440$ with assistance from R.T. L.J.R. performed phylogenetic analysis. S.H., C.H., A.L.L., and R.E.S. wrote the manuscript, and all authors approved of the final manuscript submitted.

\section{Competing interests}

The authors declare no competing interests.

\section{Additional information}

Supplementary information is available for this paper at https://doi.org/10.1038/s41467 020-18139-8.

Correspondence and requests for materials should be addressed to R.E.S. or A.L.L.

Peer review information Nature Communications thanks Edouard Jurkevitch and the other, anonymous, reviewers for their contribution to the peer review of this work. Peer reviewer reports are available.

Reprints and permission information is available at http://www.nature.com/reprints

Publisher's note Springer Nature remains neutral with regard to jurisdictional claims in published maps and institutional affiliations.

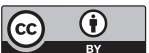

Open Access This article is licensed under a Creative Commons Attribution 4.0 International License, which permits use, sharing, adaptation, distribution and reproduction in any medium or format, as long as you give appropriate credit to the original author(s) and the source, provide a link to the Creative Commons license, and indicate if changes were made. The images or other third party material in this article are included in the article's Creative Commons license, unless indicated otherwise in a credit line to the material. If material is not included in the article's Creative Commons license and your intended use is not permitted by statutory regulation or exceeds the permitted use, you will need to obtain permission directly from the copyright holder. To view a copy of this license, visit http://creativecommons.org/ licenses/by/4.0/

(c) The Author(s) 2020 